\title{
SIMULATIONS OF HIGH-VELOCITY CLOUDS. II. ABLATION FROM HIGH-VELOCITY CLOUDS AS A SOURCE OF LOW-VELOCITY HIGH IONS
}

\author{
David B. Henley ${ }^{1}$, Kyujin KwaK ${ }^{1,2}$, and Robin L. Shelton ${ }^{1}$ \\ ${ }^{1}$ Department of Physics and Astronomy, University of Georgia, Athens, GA 30602, USA; dbh@physast.uga.edu, rls@physast.uga.edu \\ ${ }^{2}$ Korea Astronomy and Space Science Institute (KASI), 776 Daedeok-daero, Yuseong-gu, Daejeon 305-348, Republic of Korea; kkwak@kasi.re.kr \\ Received 2012 February 24; accepted 2012 April 30; published 2012 June 13
}

\begin{abstract}
In order to determine if the material ablated from high-velocity clouds (HVCs) is a significant source of low-velocity high ions ( $\mathrm{CIV}, \mathrm{Nv}$, and $\mathrm{O} \mathrm{vI}$ ) such as those found in the Galactic halo, we simulate the hydrodynamics of the gas and the time-dependent ionization evolution of its carbon, nitrogen, and oxygen ions. Our suite of simulations examines the ablation of warm material from clouds of various sizes, densities, and velocities as they pass through the hot Galactic halo. The ablated material mixes with the environmental gas, producing an intermediate-temperature mixture that is rich in high ions and that slows to the speed of the surrounding gas. We find that the slow mixed material is a significant source of the low-velocity O vI that is observed in the halo, as it can account for at least $\sim 1 / 3$ of the observed O vi column density. Hence, any complete model of the high ions in the halo should include the contribution to the O VI from ablated HVC material. However, such material is unlikely to be a major source of the observed Civ, presumably because the observed $\mathrm{C}$ IV is affected by photoionization, which our models do not include. We discuss a composite model that includes contributions from HVCs, supernova remnants, a cooling Galactic fountain, and photoionization by an external radiation field. By design, this model matches the observed O vi column density. This model can also account for most or all of the observed C IV, but only half of the observed $\mathrm{N} v$.
\end{abstract}

Key words: Galaxy: halo - hydrodynamics - ISM: clouds - methods: numerical - ultraviolet: ISM

Online-only material: color figure

\section{INTRODUCTION}

High ions from astrophysically abundant metals (e.g., C IV, $\mathrm{Nv}$, and $\mathrm{O} v \mathrm{~V}$ ) in the interstellar medium (ISM) trace spatial and temporal transitions between the hot $\left(T \gtrsim 10^{6} \mathrm{~K}\right)$ and warm/cool $\left(T \lesssim\right.$ few $\left.\times 10^{4} \mathrm{~K}\right)$ phases of the ISM. In the Galactic halo, above the disk, various physical structures may give rise to such transitions, including radiatively cooling Galactic fountain gas (Edgar \& Chevalier 1986; Shapiro \& Benjamin 1993; Benjamin \& Shapiro 1993), supernova remnants (SNRs; Shelton 1998, 2006), evaporating cold clouds that are embedded in hot ambient gas (Böhringer \& Hartquist 1987; Borkowski et al. 1990), or turbulent mixing layers formed where cool and hot gas move relative to one another (Begelman \& Fabian 1990; Slavin et al. 1993; Esquivel et al. 2006; Kwak \& Shelton 2010). Comparing the predictions of these various models with observations of high ions provides information on which physical processes are important in the Galaxy's halo.

There are plenty of observational data for such comparisons. High ions have been observed in the Galactic halo via their farultraviolet absorption lines, both at low velocities $\left(\lesssim 90 \mathrm{~km} \mathrm{~s}^{-1}\right.$; e.g., Pettini \& West 1982; Savage \& Massa 1987; Sembach \& Savage 1992; Savage et al. 1997, 2000, 2003; Zsargó et al. 2003; Indebetouw \& Shull 2004; Savage \& Wakker 2009) and high velocities ( $\gtrsim 90 \mathrm{~km} \mathrm{~s}^{-1}$; e.g., Murphy et al. 2000; Sembach et al. 2000, 2003; Fox et al. 2004, 2005, 2006; Collins et al. 2004, 2007; Shull et al. 2011). The low-velocity high ions' scale heights are $\approx 3-5 \mathrm{kpc}$ (Savage et al. 1997; Bowen et al. 2008; Savage \& Wakker 2009), although the filling factor of the high-ion-rich material is small (e.g., Shelton et al. 2007). C IV and $\mathrm{O}$ VI have also been observed in the halo via their emission lines (Shelton et al. 2001, 2007, 2010; Korpela et al. 2006; Otte \& Dixon 2006; Dixon et al. 2006; Welsh et al. 2007; Dixon \& Sankrit 2008; Park et al. 2009).

The observed ratios of high ion column densities are often used in attempts to distinguish between models (e.g., Sembach \& Savage 1992; Spitzer 1996; Sembach et al. 1997; Savage et al. 1997; Indebetouw \& Shull 2004), leading to the conclusion that a single type of model cannot explain all of the observations. However, another important consideration is the quantities of each ion that the models predict. While the normalization of many of the aforementioned models is essentially a free parameter, it is important that the normalization required by the observations is physically reasonable (for example, Indebetouw \& Shull 2004 point out that a worrisomely large number of turbulent mixing layers is needed to match the observed column densities). For other models, the normalization can be constrained in advance, without reference to the column density measurements. For example, in the model of Shelton (2006), an ensemble of SNRs above $|z|=130 \mathrm{pc}$ can account for $14 \%-39 \%$ of the typical latitude-corrected O VI column density observed toward extragalactic objects by Savage et al. (2003). In this case, the model's normalization is fixed using independently determined values for the rate and scale height of Galactic supernovae.

Here, we consider a new model: high-velocity clouds (HVCs) interacting with their surroundings. HVCs are interstellar clouds with $\left|v_{\text {LSR }}\right| \gtrsim 90 \mathrm{~km} \mathrm{~s}^{-1}$ (Wakker \& van Woerden 1997). The first HVCs were discovered via their H $21 \mathrm{~cm}$ emission (Muller et al. 1963), but are now known also to have an ionized component (e.g., Tufte 2004). On some sightlines, highly ionized high-velocity gas unassociated with high-velocity $\mathrm{H} \mathrm{I}$ is observed (e.g., Sembach et al. 2003). HVCs may be material 
in a Galactic fountain (e.g., Bregman 1980), material stripped off satellite galaxies (e.g., Gardiner \& Noguchi 1996; Putman et al. 2004), material falling into the Galaxy from extragalactic space (e.g., Oort 1966; Blitz et al. 1999), or material left over from the formation of the Galaxy (Maller \& Bullock 2004). High ions can arise from the turbulent mixing of cool cloud material with hot ambient gas (Slavin et al. 1993; Esquivel et al. 2006; Kwak $\&$ Shelton 2010; Kwak et al. 2011). There are constraints on the number of HVCs in the Galactic halo. Given these constraints, we here consider whether or not material left behind by HVCs is a significant source of the low-velocity high ions observed in the halo.

Our HVC simulations come from Kwak et al. (2011, hereafter Paper I), which used hydrodynamical simulations to study the evolution of initially spherical HVCs traveling through a hot $\left(10^{6} \mathrm{~K}\right)$ ambient medium, thought to be representative of the upper halo. Our assuming the presence of this hot gas in the halo is consistent with observations of the diffuse soft X-ray background (Burrows \& Mendenhall 1991; Wang \& Yu 1995; Pietz et al. 1998; Wang 1998; Snowden et al. 1998, 2000; Kuntz \& Snowden 2000; Smith et al. 2007; Galeazzi et al. 2007; Henley \& Shelton 2008; Lei et al. 2009; Yoshino et al. 2009; Gupta et al. 2009; Henley et al. 2010) and of absorption lines, such as O VII and $\mathrm{O}$ VIII, in the $\mathrm{X}$-ray spectra of active galactic nuclei (Nicastro et al. 2002; Fang et al. 2003; Rasmussen et al. 2003; McKernan et al. 2004; Williams et al. 2005; Fang et al. 2006; Bregman \& Lloyd-Davies 2007; Yao \& Wang 2007; Yao et al. 2008, 2009). The hot halo gas is likely due to Galactic fountains, with a possible contribution from accreting extragalactic material (Henley et al. 2010, and references therein). However, it should be noted that the distribution of this gas is not currently well known, and it need not mostly fill the halo.

In Paper I, we studied the high ions that arise when cool material ablates from an HVC and mixes with the hot ambient gas. To this end, we self-consistently traced the non-equilibrium ionization (NEI) evolution of carbon, nitrogen, and oxygen. In that paper, we concentrated on the high-velocity ions, those with line-of-sight speeds $\gtrsim 90 \mathrm{~km} \mathrm{~s}^{-1}$. We showed that the column densities and column density ratios predicted by our models overlapped those observed toward Complex C (Sembach et al. 2003; Fox et al. 2004; Collins et al. 2007). However, the highion-bearing material in our simulations eventually slows to ISMlike velocities, leading to copious quantities of low-velocity ions (with $|v| \lesssim 90 \mathrm{~km} \mathrm{~s}^{-1}$; see Section 2 for the exact definition of "low velocity" used in this paper). In this paper, we describe the evolution of these ions. We use the Galactic HVC mass infall rate to estimate the column densities of high ions in the halo expected from HVCs interacting with hot ambient gas. When we compare these predictions with observations, we find that a significant fraction ( $\gtrsim 30 \%)$ of the observed low-velocity $\mathrm{O}$ vi could be due to this process. We therefore argue that any complete model of the high ions in the halo should take into account the contribution due to HVCs.

The remainder of this paper is arranged as follows. In Section 2, we briefly describe our hydrodynamical model (see Paper I for more details). In Section 3, we describe the evolution of the low-velocity high ions, and how this is affected by our different model parameters. In Section 4, we present the average column densities of low-velocity high ions predicted by our models, and compare them with observations. In Section 5, we present $\mathrm{O}$ vi column density profiles predicted by our models. We discuss and summarize our results in Sections 6 and 7, respectively.
Table 1

Model Parameters

\begin{tabular}{|c|c|c|c|c|}
\hline $\begin{array}{l}\text { Model } \\
\text { (1) }\end{array}$ & $\begin{array}{c}r_{0} \\
(\mathrm{pc}) \\
(2)\end{array}$ & $\begin{array}{c}v_{z, \mathrm{cl}} \\
\left(\mathrm{km} \mathrm{s}^{-1}\right) \\
(3)\end{array}$ & $\begin{array}{c}n_{\mathrm{H}, \mathrm{cl}} \\
\left(\mathrm{cm}^{-3}\right) \\
(4)\end{array}$ & $\begin{array}{c}M_{\mathrm{HVC}, 0} \\
\left(M_{\odot}\right) \\
(5)\end{array}$ \\
\hline $\mathrm{A}$ & 20 & -100 & 0.1 & 120 \\
\hline B & 150 & -100 & 0.1 & $4.9 \times 10^{4}$ \\
\hline $\mathrm{C}$ & 150 & -150 & 0.1 & $4.9 \times 10^{4}$ \\
\hline D & 150 & -300 & 0.1 & $4.9 \times 10^{4}$ \\
\hline E & 150 & -150 & 0.1 & $4.9 \times 10^{4}$ \\
\hline $\mathrm{F}$ & 300 & -100 & 0.1 & $4.0 \times 10^{5}$ \\
\hline $\mathrm{G}$ & 150 & -100 & 0.01 & $4.9 \times 10^{3}$ \\
\hline
\end{tabular}

Notes. Column 1: model identifiers. Column 2: initial cloud radius. For all except Model E, this radius is approximate, as the clouds do not have sharp edges (see Figure 1 in Paper I). Column 3: initial velocity of the cloud along the $z$-direction measured in the observer's frame. Column 4: initial hydrogen number density of the cloud at its center. The ambient number density is $1 / 1000$ this value. Column 5: initial total mass of cloud, including hydrogen and helium. For all except Model E, this mass is approximate, as the clouds do not have sharp edges. Here, we use $M_{\text {init, } T}$ from Paper I-the initial mass of the material with $T<10^{4} \mathrm{~K}$

\section{HYDRODYNAMICAL MODEL}

Our hydrodynamical model is described in full in Paper I (see also Kwak \& Shelton 2010). Here, we give a brief overview of the model. The hydrodynamical simulations were carried out in two-dimensional cylindrical coordinates using FLASH version 2.5 (Fryxell et al. 2000). We tracked the ionization evolution of carbon, nitrogen, and oxygen using the FLASH NEI module. ${ }^{3}$ The simulations include radiative cooling using the default FLASH cooling curve, which is a piecewise powerlaw approximation of the Raymond \& Smith (1977) cooling curve (Rosner et al. 1978; Peres et al. 1982). This cooling curve assumes collisional ionization equilibrium (CIE); see Kwak \& Shelton (2010) for some discussion of CIE versus NEI cooling rates. Note that the model does not include a magnetic field nor thermal conduction (see Section 6.1.1).

In each simulation, the grid was initialized with a cool $\left(10^{3} \mathrm{~K}\right)$ HVC embedded in a hot $\left(10^{6} \mathrm{~K}\right)$ ambient medium. All of the clouds are initially spherical. The density of hydrogen atoms in each cloud $\left(n_{\mathrm{H}, \mathrm{cl}}\right.$ in Table 1$)$ pertains to the density of the cloud interior. Near the cloud's periphery, the density decreases smoothly to that of the ambient gas $\left(n_{\mathrm{H}, \mathrm{amb}}=n_{\mathrm{H}, \mathrm{cl}} / 1000\right)$, and the temperature increases smoothly from $10^{3}$ to $10^{6} \mathrm{~K}$, except in Model E, which has a sharp edge (see Figure 1 in Paper I). Initially, the cloud interior, ambient medium, and transition zone are in pressure balance. Note that, although the densities are expressed in terms of the hydrogen number density, the gas also includes helium $\left(n_{\mathrm{He}} / n_{\mathrm{H}}=0.1\right)$, so the total number density of atoms and ions is $1.1 n_{\mathrm{H}}$. We assume that hydrogen and helium are fully ionized at all temperatures, so the electron density $n_{\mathrm{e}}=1.2 n_{\mathrm{H}}$.

The simulations were carried out in the initial rest frame of the HVC; i.e., the HVC was initially at rest, while the ambient medium moved upward with velocity $\left|v_{z, \text { cl }}\right|$, where $v_{z, \text { cl }}$ is the HVC's initial velocity in the observer's frame (the observer is assumed to be located below the domain, looking vertically upward). The boundary conditions allowed material to flow in

\footnotetext{
3 As noted in Paper I, we do not include the important high ion Si IV because it is more susceptible to photoionization. Modeling photoionization is beyond the scope of this paper.
} 
from the bottom of the domain and flow off the top of the domain. Before extracting masses or column densities of high ions as a function of velocity, we transformed the velocities to the observer's frame.

The parameters for each model in our suite of models are listed in Table 1. Model B is our reference model. The other models allow us to investigate the effects of cloud size (Models A, B, and F), cloud velocity (Models B, C, and D), cloud density (Models B and G), and cloud density profile (Models C and E).

In the following, we distinguish between high- and lowvelocity ions using the same cuts in velocity that we used in Paper I. In Models A, B, F, and G (in which the initial velocity of the cloud was $v_{z, \mathrm{cl}}=-100 \mathrm{~km} \mathrm{~s}^{-1}$ ), material with $v_{z} \leqslant-80 \mathrm{~km} \mathrm{~s}^{-1}$ is defined as high velocity, while that with $v_{z}>-80 \mathrm{~km} \mathrm{~s}^{-1}$ is defined as low velocity, where $v_{z}$ is the $z$-velocity of the gas in the observer's frame. For Models C, D, and $\mathrm{E}$ (in which $v_{z, \mathrm{cl}}$ was -150 or $-300 \mathrm{~km} \mathrm{~s}^{-1}$ ), the velocity cut is at $v_{z}=-100 \mathrm{~km} \mathrm{~s}^{-1}$.

When calculating the quantities of high ions that result from the cloud-ISM interactions, we assumed the Wilms et al. (2000) interstellar abundances for carbon, nitrogen, and oxygen. Note that in Paper I we used older abundances, from Allen (1973).

\section{EVOLUTION OF LOW-VELOCITY HIGH IONS}

The hydrodynamical interaction between the model HVCs and the ambient gas is described in detail in Paper I. We begin this section by giving an overview of the processes. As a model HVC moves through the ISM, material is ablated from the cloud. The cool ablated material mixes with the hot ambient gas, creating gas of intermediate temperature $\left(T \sim(1-3) \times 10^{5} \mathrm{~K}\right)$ in which high ions are abundant. The temperature of this mixed gas is affected both by radiative cooling and continued mixing with the hot ambient gas. The fractions of high ions in the mixed gas increase both by ionization of the initially cool ablated material and by recombination of the initially hot ambient material. However, the fractions of all ions differ by varying degrees from those expected from CIE, as changes in the ionization balance lag behind the changes in the gas temperature that result from mixing and radiative cooling. Soon after material is torn from the clouds, it becomes relatively rich in high ions. At that time, the material is traveling almost as fast as the HVC, but as the ablated material continues to mix with the ambient medium, it slows. This causes the velocities of the ions to tend toward that of the ISM, and the ablated material to drift further from the cloud.

In Figure 1, we show where the low-velocity ions are located relative to the HVC, by plotting the mass of each ion as a function of height. These plots were created using data from a modified version of Model $\mathrm{B}$ called Model $\mathrm{B}_{\mathrm{ext}}$, in which the domain extends up to $z_{\max }=4400 \mathrm{pc}$, instead of $z_{\max }=2800 \mathrm{pc}$ as in the original Model B (we increased $z_{\max }$ for this purpose so we could trace the high ions over a greater range of heights). The height, $z$, is measured in the frame in which the HVC was initially at rest at $z=0$. The HVC shifts upward during the course of the simulation, due to the ram pressure of the ambient medium. However, as this shift is only $\sim 200$ pc by $t=120 \mathrm{Myr}, z$ is approximately the height above the HVC's current position. We show results for two different epochs, $t=$ 60 and $120 \mathrm{Myr}$. In each panel, the black curve shows the results of the NEI calculations, and the gray curve shows the results obtained assuming CIE, where the ion fractions depend only on the local gas temperature. Note that, in general, there are significantly more high ions than are expected from CIE. Note also that the peaks seen at the two epochs do not represent the same ions - the peaks move through the domain at $\sim 80-100$ $\mathrm{km} \mathrm{s}^{-1}$ and so would move $\sim 5-6 \mathrm{kpc}$ in $60 \mathrm{Myr}$.

We see that the low-velocity C IV generally resides within $1400 \mathrm{pc}$ of the cloud (for both CIE and NEI predictions) at both 60 and 120 Myr. On average, both the N v and the O vI reside further behind the cloud. This is as expected-the mixed gas further behind the cloud contains a larger fraction of initially ambient gas and so, ignoring the effects of radiative cooling for the moment, is hotter and more highly ionized than that nearer to the cloud.

Eventually, near the top of the domain, the mixed gas generally starts becoming too hot even for $\mathrm{O}$ VI, due to the continued mixing of the ablated cloud material with the hot ambient gas. This continued mixing and heating appears to be the ultimate fate for most of the ablated material-by the end of Model B, $38 \%$ of the material that was initially in the cloud and is now above $z=2 \mathrm{kpc}$ is hotter than $6 \times 10^{5} \mathrm{~K}$, while only $13 \%$ of this material is cooler than $1 \times 10^{5} \mathrm{~K}$. The fraction of the initial cloud material that is hotter than $6 \times 10^{5} \mathrm{~K}$ by the end of Model B increases with increasing $z$. The hot mixed gas also becomes more tenuous, because of the low density of the ambient gas. Both of these effects lead to a fall off in the number of high ions. In addition, the mixed gas is closer to, although not yet in, equilibrium.

Although most of the mixed gas is hot, it was noted in Paper I that radiatively cooled mixed gas $\left(T \lesssim 10^{4} \mathrm{~K}\right)$ accumulates along the symmetry $(r=0)$ axis of the domain. This radiatively cooled gas may be observable (e.g., via $\mathrm{H} \alpha$ emission), although such predictions are beyond the scope of this paper. However, it should be noted that this accumulation of cooled gas along the symmetry axis may be an artifact of the cylindrical geometry. Furthermore, because of the cylindrical geometry, this cooled gas represents a small fraction of the mixed gas mass. This also means that any further mixing of this cooled gas with hotter gas makes a negligible contribution to the total high ion content.

It is useful to calculate the time evolution of the numbers of or masses of low-velocity high ions that result from the cloud-ISM interaction. Summing the amounts of such ions in the model domain at a given epoch provides one measure, but the masses of the ions in the domain are lower limits on the true masses of the ions that result from the HVC-ISM interaction. This is because these masses do not take into account material that has flowed out of the model domain (recall that each simulation is carried out in the cloud's rest frame and that material is allowed to flow off the domain at the top boundary).

For most models, we can make an estimate of the upper limit on a given ion's mass by including material that has flowed off the domain. We are able to estimate the quantity of material that leaves the domain between each epoch in the model (i.e., the times at which the hydrodynamical data are output from the code) from the vertical velocity and position of the cells near the top of the domain. Gas that is traveling upward at velocity $v$ and that is a distance $<v \Delta t$ from the top of the domain at the current epoch will leave the grid by the next epoch, where $\Delta t$ is the time between epochs (e.g., $0.5 \mathrm{Myr}$ for Model B; note that $\Delta t$ does not refer to the simulational time step, which is much shorter). We record the mass of each high ion that passes beyond the top of the domain at each epoch.

Clearly, we cannot trace the evolution of the escaping material after it has left the computational domain, but we can tally the amount of a given high ion that has crossed the upper 

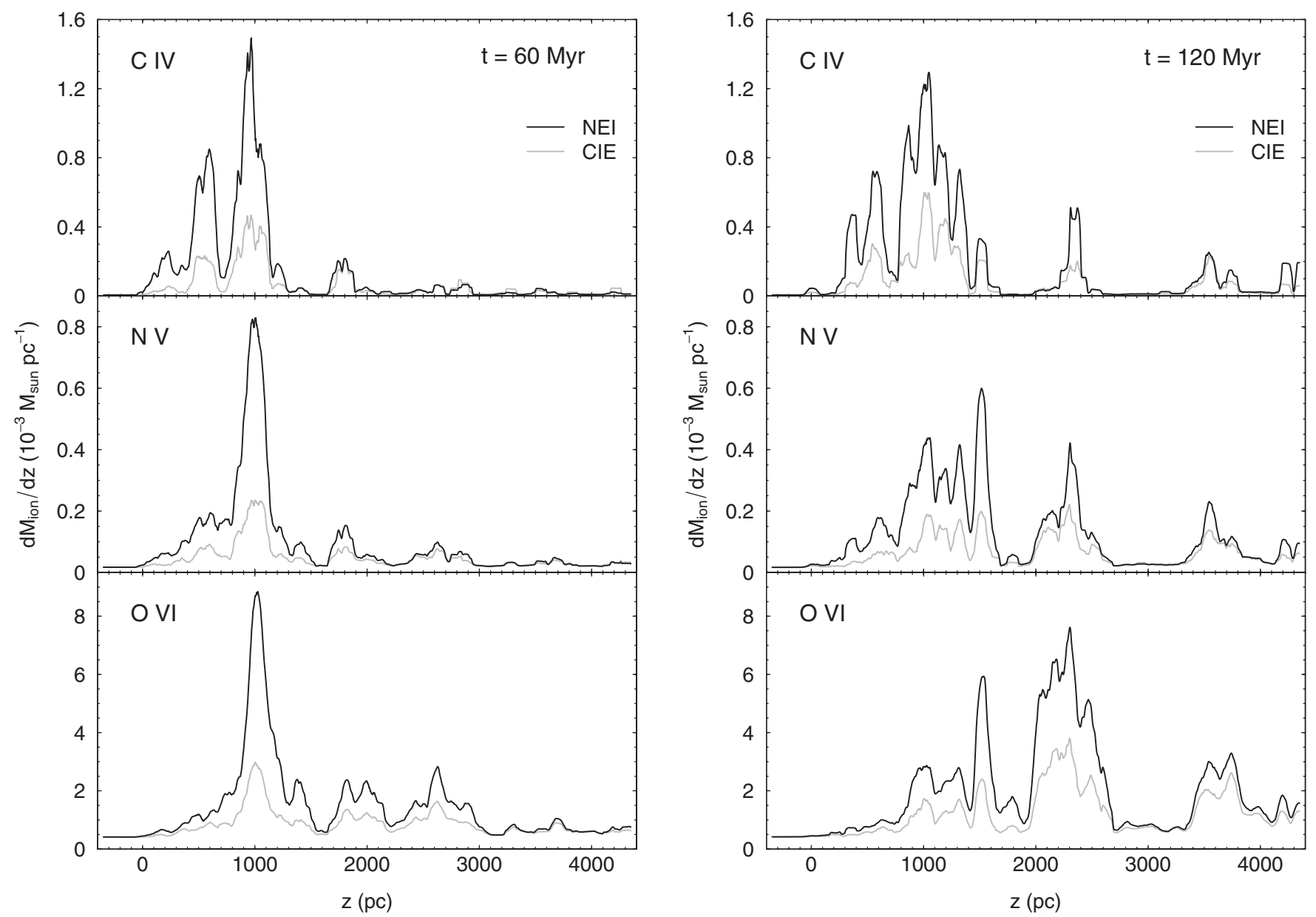

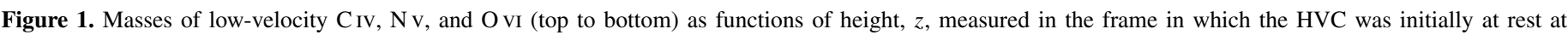

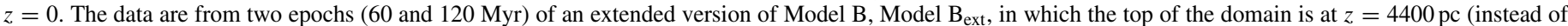

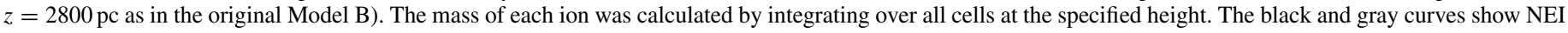
and CIE results, respectively. The data have been smoothed with a boxcar of full width $100 \mathrm{pc}$.

domain boundary at all preceding times. This tally serves as an upper limit on the amount of that ion beyond the upper boundary at any given time, because it ignores the possibility that some of the escaped material further ionizes (as would occur when the gas mixes with additional hot ambient gas, raising the temperature above that which is favorable to $\mathrm{C} \mathrm{IV}, \mathrm{N} \mathrm{V}$, and $\mathrm{O}$ VI) or recombines. The sum of the amount of a given high ion that has moved out of the domain and the amount currently in the domain forms our upper limit on the total amount of that high ion present.

Using Model $\mathrm{B}_{\mathrm{ext}}$, we investigated the evolution of the lowvelocity high ions after they have risen above $z=2800 \mathrm{pc}$, the maximum height in Model B. We do this by following the time evolution of the ion masses contained in selected peaks in the Model $B_{\text {ext }}$ mass-versus-height distribution (e.g., Figure 1) as these peaks drift beyond the height of Model B. Figure 2, for example, shows the masses of $\mathrm{O}$ VI and $\mathrm{C}$ IV in a peak that we traced from $t=72$ to $90 \mathrm{Myr}$, during which time it drifted from $z \approx 2000$ to $\approx 3600 \mathrm{pc}$. Both the mass of O VI and the mass of $\mathrm{C}_{\mathrm{IV}}$ in the peak decreased during this time (as did that of $\mathrm{N} v$, not shown). Assuming that this trend applies to all of the low-velocity high ions that rise past $z=2800 \mathrm{pc}$ in Models B and $B_{\text {ext }}$, the tally of all low-velocity high ions that have passed the upper boundary in Model B by some epoch of interest is an upper limit on the mass of high ions above the boundary at that

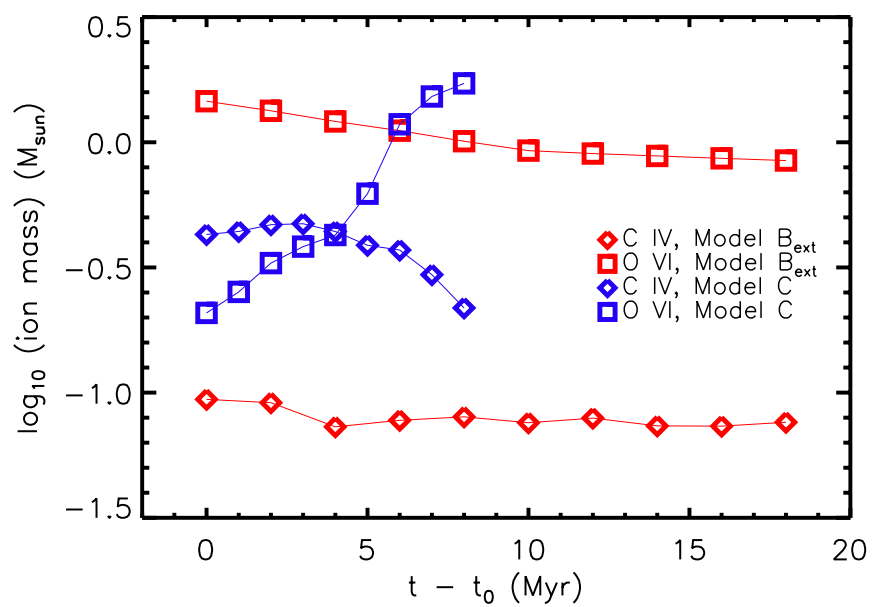

Figure 2. Masses of low-velocity C IV (diamonds) and O vi (squares) in a single peak from the ion mass vs. height distributions (Figure 1) as functions of time. The Model $\mathrm{B}_{\mathrm{ext}}$ data (red) are from a peak that we traced from $t=t_{0}=72$ to $t=90 \mathrm{Myr}$, during which time it moved from $z \approx 2000$ to $z \approx 3600 \mathrm{pc}$. The ion masses include all ions within $\Delta z= \pm 250 \mathrm{pc}$ of the peak. The Model C data (blue) are from a peak that we traced from $t=t_{0}=99$ to $t=107 \mathrm{Myr}$, during which time it moved from $z \approx 1300$ to $z \approx 2500 \mathrm{pc}$. The ion masses include all ions within $\Delta z= \pm 150 \mathrm{pc}$ of the peak.

(A color version of this figure is available in the online journal.) 
epoch. We expect this also to be true for the other models with $v_{z, \mathrm{cl}}=-100 \mathrm{~km} \mathrm{~s}^{-1}$ (Models A, F, and G).

In models with $\left|v_{z, \mathrm{cl}}\right|>100 \mathrm{~km} \mathrm{~s}^{-1}$, the ram pressure of the ambient medium pushes the cloud upward during the course of the simulation (see Figure 4 in Paper I). As a result of this shift and the greater flow speed in the ambient medium, in the later stages of the simulation ablated material leaves the domain soon after it is torn from the HVC. The fractions of carbon, nitrogen, and oxygen in the $\mathrm{C} \mathrm{IV}, \mathrm{N} \mathrm{v}$, and $\mathrm{O}$ vi ionization levels, respectively, may still be increasing in this outflowing material when it leaves the domain. Also, in Model D in particular, a large fraction of the high ions that flow off the domain do so at high velocities. This high-velocity material may slow to low velocities after leaving the domain while remaining rich in high ions. Thus, it is possible that the conclusion drawn from Model $\mathrm{B}_{\text {ext }}$, above, does not apply to Models C, D, and E. We found, for example, by examining a Model $\mathrm{C}$ mass peak that the quantity of $\mathrm{O}$ VI ions was still increasing as the material approached the upper boundary of the domain (see Figure 2), raising the possibility that it would have continued to increase with time after crossing the $z=2800 \mathrm{pc}$ mark, had the simulation domain included greater heights. This was not the case for $\mathrm{C}$ IV or $\mathrm{N} \mathrm{v}$, whose masses had already begun to decrease while the mass peak was still in the domain (see Figure 2 for C IV). Hence, for Models C, D, and E, taking into account material that has left the domain may not always yield upper limits on the true total masses of the high ions.

Figures 3-5 show the lower and upper limits on the masses of $\mathrm{C}$ IV, $\mathrm{N}$ v, and O VI, respectively, that result from the HVC-ISM interaction. These masses are plotted as functions of time for each of our seven hydrodynamical models. We plot the masses of both low-velocity and high-velocity ions (black and gray lines, respectively). In each case, we plot the mass of the ion in the domain (dashed line), and that mass plus the mass of the ion in all the material that has ever escaped from the domain (solid line). As noted previously, the former is the lower limit on the true mass, while the latter is our best estimate of the upper limit.

\subsection{General Features in the Evolution of the Low-velocity High Ions}

Here, we discuss the evolution of the low-velocity high ions in our reference model, Model B, and point out features that are common across all or most of the other models. In the following subsection, we will discuss how the different model parameters affect the evolution of these ions.

The number of low-velocity high ions generally increases with time throughout the simulations. Admittedly, the masses of the high ions in the domain are decreasing toward the end of the Model B simulation. However, as $\sim 70 \%$ of the cloud's initial H I mass remains at the end of the simulation (Paper I), it is likely that this decrease is due to a relatively short-term fluctuation in the number of high ions, rather than due to a longterm decline in the processes that lead to high-ion-rich material. However, if we could run the simulation to much later times, we would eventually exhaust the cloud, and the processes that lead to high-ion-rich material would decline and eventually cease. The number of high ions would then decrease to zero as the high-ion-bearing gas either radiatively cooled or mixed with the million-degree ambient gas.

When we compare the masses of low-velocity ions in Model B that do and do not take into account material that has escaped from the domain (solid and dashed black curves, respectively),
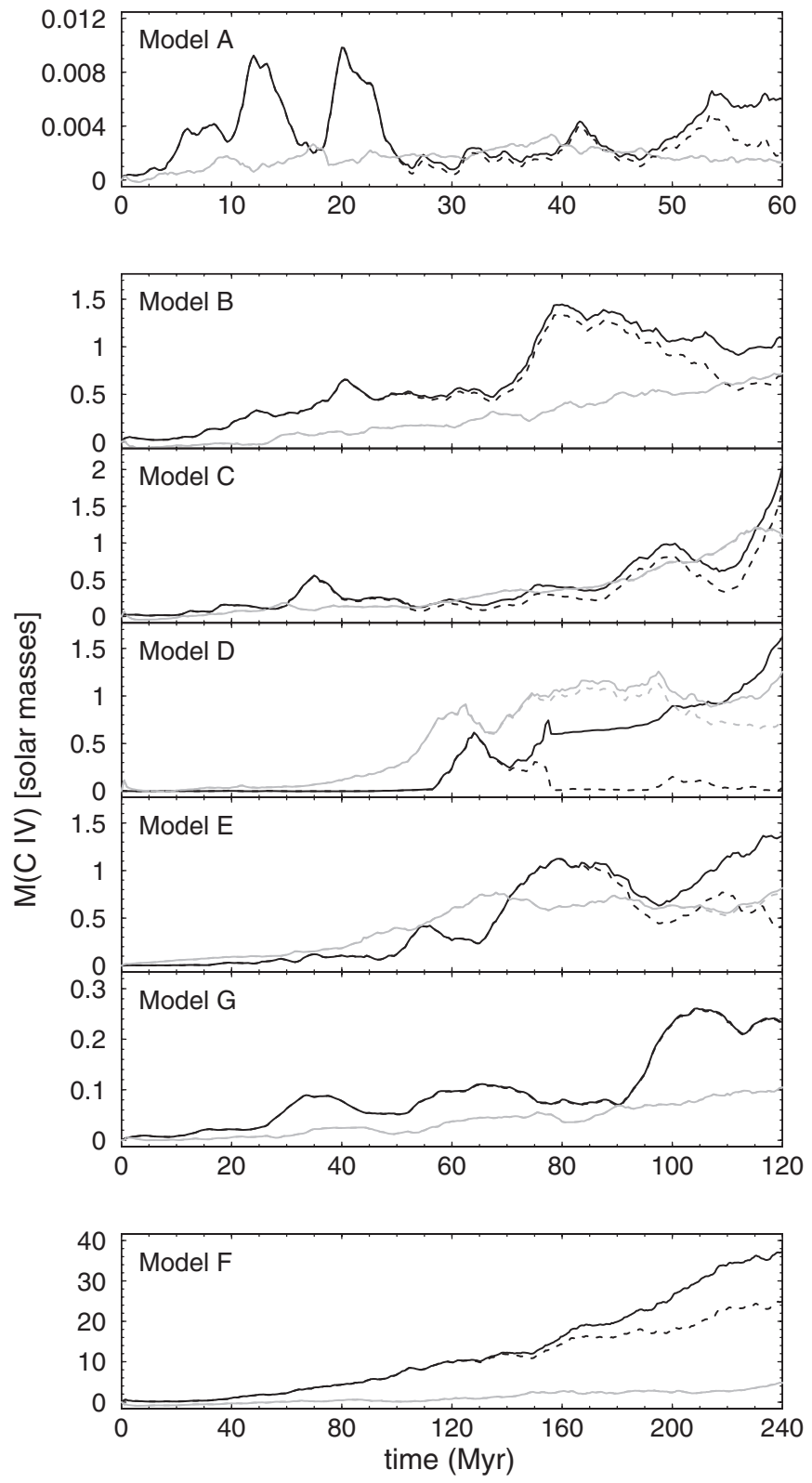

Figure 3. Mass of $\mathrm{C}$ IV as a function of time from our seven hydrodynamical models (note the different ranges on the time axes). In each panel, the black lines show the masses of low-velocity ions, and the gray lines show the masses of high-velocity ions. The dashed lines show the mass of each ion that is in the model domain, while the solid lines include ions that have escaped from the top of the domain (see the text for details). Note that, in most panels, the gray solid and dashed lines are indistinguishable.

we see that these lines diverge by greater amounts as we go from C IV to $\mathrm{N} v$ to $\mathrm{O}$ VI (Figures 3, 4, and 5, respectively). This behavior is understandable from Figure 1. The low-velocity $\mathrm{C}$ IV mainly resides close to the $\mathrm{HVC}$, well away from the top of the domain, and so very little low-velocity $\mathrm{C}$ IV flows off the domain. In contrast, the low-velocity $\mathrm{O}$ vi extends further behind the HVC, nearer the top of the domain, and so significant quantities of this ion escape from the domain. This general behavior is seen in the other models. However, it should be noted that in Model $\mathrm{D}$ the ambient material passes through the domain two or three times faster than in the other models. As a result, the mixed C IV-bearing gas is swept off the grid by the fast flow of the ambient gas. The fact that significant quantities of low-velocity 

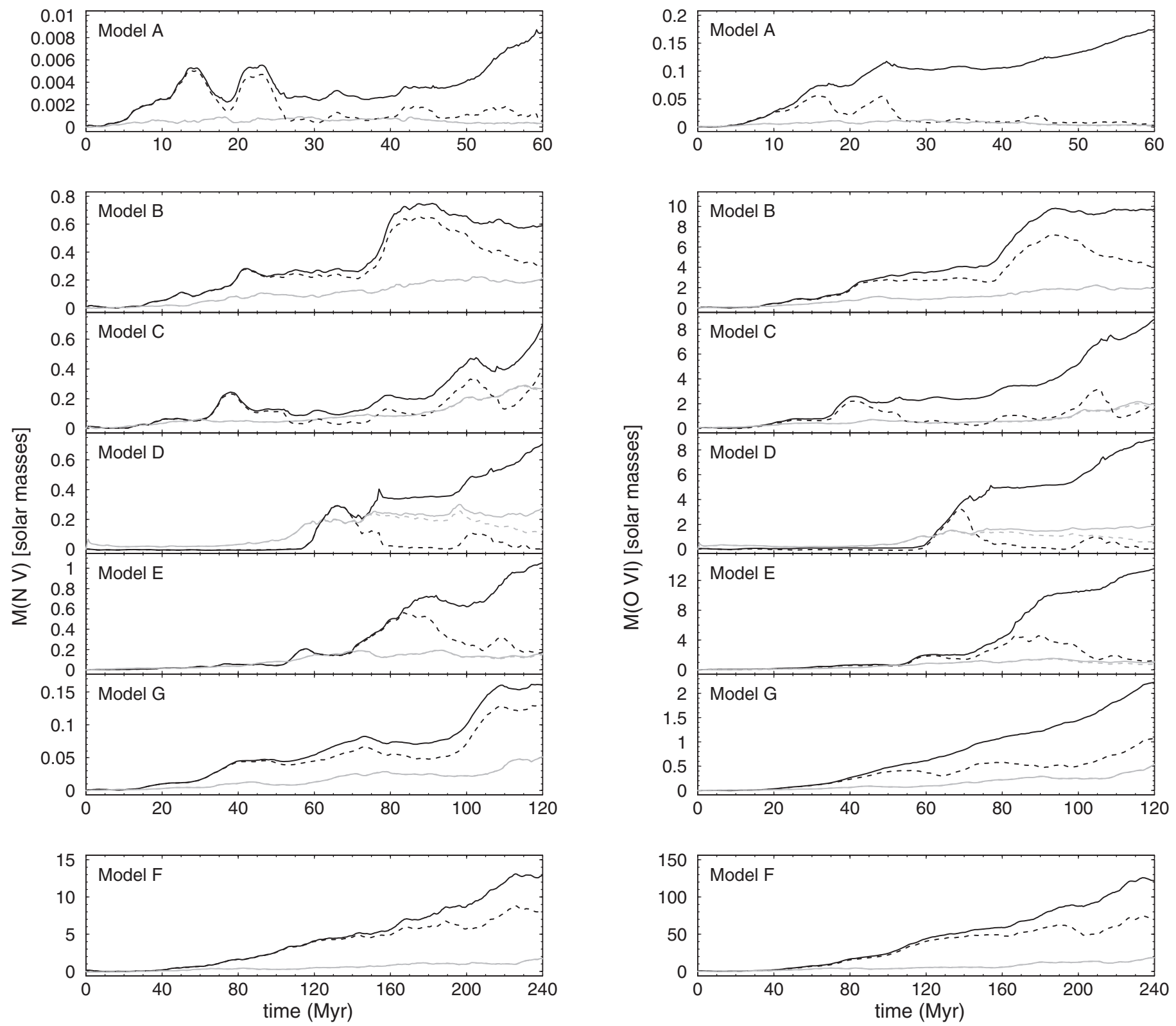

Figure 4. Same as Figure 3, but for N v.

C IV are lost from the domain in this simulation is reflected by the wide gap between the solid and dashed black curves in the Model D panel of Figure 3.

We note that, in most cases, most of the high ions are at low velocities, despite their origin in the interaction of an HVC with its surroundings. Although we are concentrating on the low-velocity ions here, we point out that the solid gray (highvelocity material including that which has left the domain) and dashed gray (high-velocity material in the domain) curves in Figures 3-5 are indistinguishable in nearly every panel, implying that virtually no high-velocity high ions escape from the domain (i.e., the ions slowed to low velocities before they left the domain). ${ }^{4}$ Again, the exception to this is Model D.

\footnotetext{
4 We remind the reader that "low velocity" and "high velocity" refer to velocities in the observer's frame, whereas the simulations were carried out in the HVC's initial rest frame. Hence, "low-velocity" ions are moving quickly in the simulation domain, whereas "high-velocity" ions move relatively slowly.
}

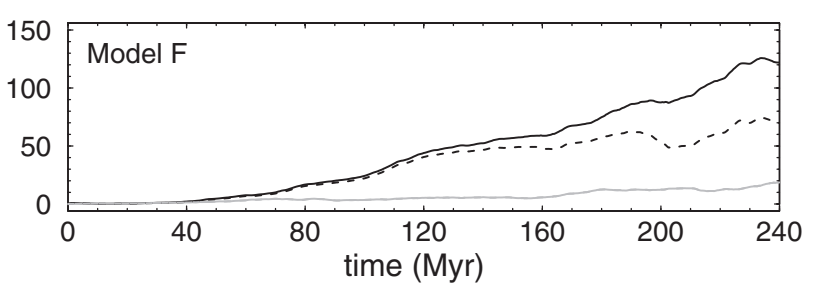

Figure 5. Same as Figure 3, but for O VI.

\subsection{Effects of the Cloud Velocity, Profile, Density, and Size}

Cloud velocity. Models B, C, and D have identical initial clouds and ambient gas, but differing initial cloud velocities $\left(-100,-150\right.$, and $-300 \mathrm{~km} \mathrm{~s}^{-1}$, respectively), allowing us to examine the effect of cloud velocity. As noted in Paper I, these velocities correspond to the subsonic, transonic, and supersonic regimes, respectively, resulting in significant differences in the morphological evolution of the HVCs.

One key difference between the models is that in Model D a bow shock forms in front of the cloud, which helps protect the cloud from ablation at earlier times and delays the onset of mixing (Paper I). As a result, there are very few high ions (high or low velocity) in Model D before $t \sim 40 \mathrm{Myr}$. Another key difference is that the fast-moving ambient medium in Model $\mathrm{D}$ tends to sweep high ions off the domain before they are able to slow to low velocities in the observer's frame. As a result, although high-velocity ions start becoming abundant in 
Model D at $t \sim 40 \mathrm{Myr}$, low-velocity ions do not become abundant until $t \sim 60 \mathrm{Myr}$. During this time delay, the HVC and hence the material ablated from it decelerated sufficiently for the mixed, high-ion-rich gas to reach low velocities before leaving the domain.

After $t \sim 60 \mathrm{Myr}$, despite the differences in the clouds' morphological evolution, the masses of low-velocity high ions that include the ions that have escaped from the domain (solid black curves in Figures 3-5) agree within a factor of $\sim 3$ for all three models. If we consider only the ions in the domain (dashed black curves in Figures 3-5), we see that Model D has far fewer high ions than Model B after $t \sim 80 \mathrm{Myr}$. This is likely because the Model D HVC shifts upward in the domain during the course of the simulation, due to the large ram pressure of the ambient medium (see Figure 4 in Paper I). Eventually, the HVC gets so close to the top of the domain that, once again, the high ions are unable to slow to low velocities before leaving the domain.

When considering the difference between the solid and dashed black curves in Figures 3-5, Model C generally lies between Models B and D. As in Model D, the Model C cloud shifts upward in the domain during the simulation. Because the Model C cloud gets closer to the top of the domain, relatively more low-velocity high ions are swept off the top of the domain than in Model B. This has the greatest effect on $\mathrm{O}$ VI, as $\mathrm{O}$ VI tends to exist further behind the HVC than $\mathrm{C}$ IV or $\mathrm{N} \mathrm{v}$. Model E, in which the cloud velocity is the same as in Model $\mathrm{C}$, is similarly affected.

Cloud density profile. The only difference between the initial parameters in Models C and E is the HVC's initial density profile (smooth-edged versus sharp-edged; see Figure 1 in Paper I). The large density contrast at the edge of the Model E cloud inhibits the growth of shear instabilities and delays the onset of mixing, resulting in fewer high ions than in Model C. However, after $t \sim 50 \mathrm{Myr}$, the predictions from these models that include the ions that have escaped from the domain generally agree within a factor of $\sim 2$, with Model E tending to yield larger masses.

Cloud density. The only differences between Models B and $\mathrm{G}$ are that the cloud and ambient densities are 1/10 as large in Model $\mathrm{G}$ than in Model B. As expected, the lower densities in Model $\mathrm{G}$ result in fewer high ions. However, there are more high ions in Model $G$ than one would expect from a simple rescaling of the Model B results. This difference is related to the fact that radiative cooling operates at a slower rate in Model $\mathrm{G}$, because of the lower density. This difference in cooling rate affects the temperature distribution in the gas, which in turn affects the ionization and recombination rates. Nevertheless, despite these differences, the ion masses in Models B and G generally agree within a factor of $\sim 2$, after allowing for the factor of 10 difference in density.

Cloud size. Models A, B, and F differ by the HVC's initial radius $\left(r_{0}=20,150\right.$, and $300 \mathrm{pc}$, respectively) and hence initial $\operatorname{mass}\left(M_{\mathrm{HVC}, 0}=120,4.9 \times 10^{4}\right.$, and $4.0 \times 10^{5} M_{\odot}$, respectively $)$. Unsurprisingly, the more massive the HVC, the greater the mass of low-velocity ions that results from its interaction with its surroundings.

We can examine this behavior more quantitatively by using a simple model of a uniform spherical HVC shedding mass at a rate proportional to its surface area (Paper I, Section 3.3.3). In this simple model, we expect the mass of a given low-velocity ion, $M_{\text {ion }}$, at a rescaled simulation time of $t / r_{0}$, to be proportional to the cloud's initial mass, $M_{\mathrm{HVC}, 0}$ (see Equation (A2) in the Appendix). The rescaling of the simulation time by dividing by $r_{0}$ is necessary if we wish to compare models of different-sized
HVCs at equivalent stages in their evolution, in the sense of their having had the same fraction of their initial H I mass ablated (see Paper I). Note that, in deriving $M_{\text {ion }}\left(t / r_{0}\right) \propto M_{\mathrm{HVC}, 0}$, we have ignored the fact that the ions of interest will subsequently recombine or ionize. While this is a gross approximation, this simple model does provide some insight into the relative behaviors of Models A, B, and F.

We find that the scaling of $M_{\text {ion }}$ with $M_{\mathrm{HVC}, 0}$ from our hydrodynamical models is close to the scaling expected from this simple model. When we compare Models B and A, we find $M_{\text {ion }}\left(t / r_{0}\right) \propto M_{\mathrm{HVC}, 0}^{\gamma}$, with $\gamma \approx 0.8-1.0$ (the exponent varies with $t / r_{0}$ and with ion). When we compare Models $\mathrm{B}$ and $\mathrm{F}$, we find that $\gamma$ tends to be somewhat larger: $\gamma \approx 0.9-1.6$. (In these comparisons, we used the ion masses that include material that has escaped from the domain, and we ignored early times in the simulations $\left(t / r_{0}<0.2 \mathrm{Myr} \mathrm{pc}^{-1}\right.$ or $t<4$, 30, and 60 Myr for Models A, B, and F, respectively).) Thus, the values of $M_{\text {ion }}\left(t / r_{0}\right)$ scale approximately as expected with $M_{\mathrm{HVC}, 0}$. The deviations from $M_{\mathrm{ion}}\left(t / r_{0}\right) \propto M_{\mathrm{HVC}, 0}$ may be due to our neglecting the recombination or ionization of the high ions.

At later times in Model A, the masses of low-velocity $\mathrm{N} \mathrm{V}$ and $\mathrm{O}$ VI that do and do not take into account material that has left the domain (black solid and black dashed lines, respectively) start to diverge significantly. This divergence is due to an effect already mentioned for Models $\mathrm{C}$ and $\mathrm{E}$ above: the cloud shifts upward in the model domain, leading to more low-velocity high ions being swept off the top of the domain. In the case of Model A, it is the cloud's low mass-to-cross-section ratio that makes it susceptible to being pushed upward by the ambient medium's ram pressure.

\section{COMPARISON WITH OBSERVATIONS: THE HALO COLUMN DENSITY OF LOW-VELOCITY HIGH IONS}

\subsection{Model Column Density Predictions}

In order to compare the results presented in the previous section with observations, we must calculate from these results the expected column densities of low-velocity high ions. In order to do this, we assume that the observed population of HVCs can be modeled by some number of model clouds, each of which is similar to one of our simulated clouds. In this case,

$$
\mathcal{N}_{\mathrm{HVC}}=\frac{\dot{\mathcal{M}}_{\mathrm{HVC}}^{\mathrm{H}} T_{\mathrm{HVC}}}{M_{\mathrm{HVC}, 0}^{\mathrm{HI}}}
$$

is the number of model HVCs needed to account for the mass of high-velocity material, where $T_{\mathrm{HVC}}$ and $M_{\mathrm{HVC}, 0}^{\mathrm{HI}}$ are the lifetime and initial $\mathrm{HI}$ mass $^{5}$ of the model cloud, respectively, and $\dot{\mathcal{M}}_{\text {HVC }}^{\mathrm{H}}$ is the observed infall rate of HVCs in the Galactic halo, expressed in terms of the total (neutral + ionized) hydrogen mass. By using an observed HVC infall rate that includes both neutral and ionized material, we are implicitly assuming that the observed HVCs began their lives as entirely neutral material or, if they were initially partially ionized, that this does not have a significant effect on the quantities of high ions that result from their mixing with the hot halo.

If $\left\langle M_{\mathrm{ion}}\right\rangle_{\mathrm{HVC}}=\int M_{\mathrm{ion}}(t) d t / T_{\mathrm{HVC}}$ is the time-averaged total mass of a given low-velocity ion due to ablation from a single model HVC, then the total mass of that ion in the halo due to

\footnotetext{
5 Note that the cloud masses in Table 1 are total masses, and so must be divided by 1.4 to give the $\mathrm{H}$ I masses.
} 
ablation from the population of HVCs is given by

$$
\begin{aligned}
M_{\text {ion, halo }} & =\left\langle M_{\mathrm{ion}}\right\rangle_{\mathrm{HVC}} \mathcal{N}_{\mathrm{HVC}} \\
& =\frac{\dot{\mathcal{M}}_{\mathrm{HVC}}^{\mathrm{H}}}{M_{\mathrm{HVC}, 0}^{\mathrm{HI}}} \int M_{\mathrm{ion}}(t) d t .
\end{aligned}
$$

If we assume that these ions are uniformly distributed in a cylindrical halo of radius $R_{\mathrm{MW}}$ above and below the disk, the average column density for a vertical sightline is

$$
\bar{N}(\text { ion })=\frac{\dot{\mathcal{M}}_{\mathrm{HVC}}^{\mathrm{H}}}{2 \pi R_{\mathrm{MW}}^{2} M_{\mathrm{HVC}, 0}^{\mathrm{HI}} m_{\mathrm{ion}}} \int M_{\mathrm{ion}}(t) d t,
$$

where $m_{\text {ion }}$ is the atomic mass of the ion $(12.011,14.00674$, and $15.9994 \mathrm{u}$ for $\mathrm{C} \mathrm{IV}, \mathrm{N} \mathrm{v}$, and O VI, respectively (taken from the FLASH code)).

The value of the HVC infall rate, $\dot{\mathcal{M}}_{\mathrm{HVC}}^{\mathrm{H}}$, is uncertain. If the observed HVCs are accreting extragalactic material, estimates for $\dot{\mathcal{M}}_{\mathrm{HVC}}^{\mathrm{H}}$ range from $\sim 0.2 M_{\odot} \mathrm{yr}^{-1}$ (Mirabel 1989; Peek et al. 2008) to $\sim 1 M_{\odot} \mathrm{yr}^{-1}$ (Wakker \& van Woerden 1997). If the observed HVCs are mainly due to a Galactic fountain rather than due to accretion, there may be up to $\sim 5 M_{\odot} \mathrm{yr}^{-1}$ circulating through the halo (Wakker \& van Woerden 1997). We assume $\dot{\mathcal{M}}_{\mathrm{HVC}}^{\mathrm{H}}=0.5 M_{\odot} \mathrm{yr}^{-1}$. Considering that infall rates for individual complexes are as large as $\sim 0.1 M_{\odot} \mathrm{yr}^{-1}$ for Complex C (Wakker et al. 2007; Thom et al. 2008), $\gtrsim 0.1 M_{\odot}$ $\mathrm{yr}^{-1}$ for the Smith Cloud (using data from Lockman et al. 2008 and Hill et al. 2009), and $\sim 0.1-0.5 M_{\odot} \mathrm{yr}^{-1}$ for the Magellanic Stream (the lower value is estimated from the mass of the negative-velocity portion of the Stream from Brüns et al. 2005; the upper value is from Mirabel 1989), our assumed value of $\dot{\mathcal{M}}_{\mathrm{HVC}}^{\mathrm{H}}$ should be reasonably conservative. We also take $R_{\mathrm{MW}}=25 \mathrm{kpc}$ (Ferrière 2001).

Ideally, the time integration of $M_{\text {ion }}(t)$ in Equation (3) should be carried out over the entire lifetime of the high ions in the halo. Note that this lifetime is, in general, not the time it would take for the HVC to be disrupted in the halo, because (a) the low-velocity high ions would tend to persist after the $\mathrm{H}$ I HVC has been completely disrupted or (b) the HVC may reach the disk before it has been completely disrupted. In practice, if we integrate $M_{\text {ion }}(t)$ up to the end of our simulations, then we obtain a lower limit on $\int M_{\mathrm{ion}}(t) d t$, as we are neglecting the contributions from beyond the ends of the simulations. In this subsection, we present the column density predictions that result from the simulated part of the HVC evolution. In Section 4.3, below, we describe a method for including the estimated contributions from beyond the ends of the simulations.

The column densities calculated using Equation (3) up to the times when the simulations ended are shown in the upper half of Table 2 and are plotted in black in Figure 6. In each case, we have calculated the column density using only the ions that are in the simulational domain ("Domain only"), and using the ions in the domain plus those that have escaped ("Domain + Escaped"). For Model A, we also tabulate column densities resulting from only the first $16 \mathrm{Myr}$ of the simulation $\left(\mathrm{A}^{*}\right.$ in Table 2; see Section 4.2). Note in particular that the Domainonly values are strict lower limits on the true model predictions (i.e., the column densities that the models would predict if we could trace all of the high ions indefinitely). This is because the mass of an ion within the simulation domain is a lower limit on the true value of $M_{\text {ion }}(t)$, and so each Domain-only column density is calculated from the lower limit of the integral of a quantity that is itself a lower limit.
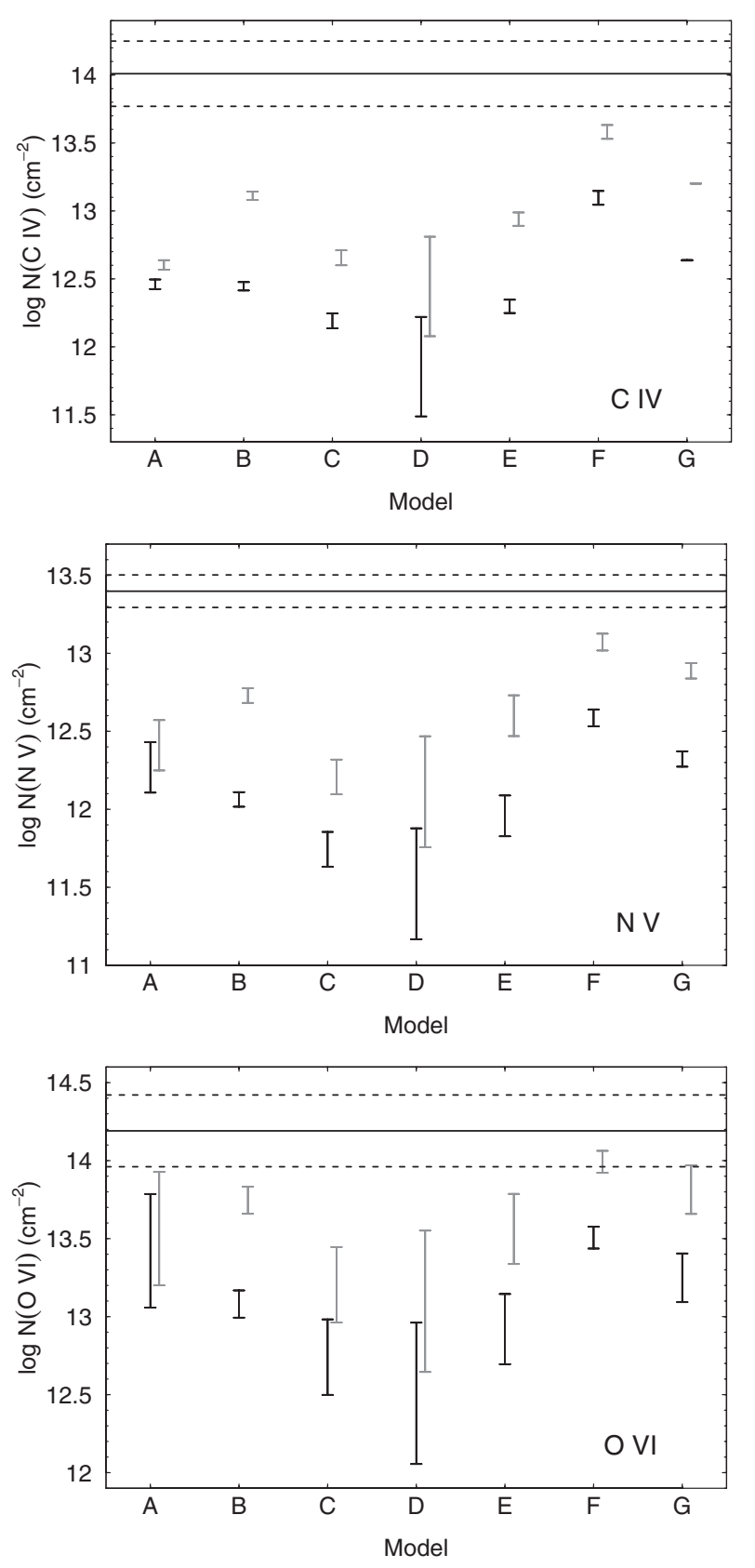

Figure 6. Comparison of the observed and predicted low-velocity halo column densities for $\mathrm{CIV}, \mathrm{Nv}$, and $\mathrm{O}$ VI (top to bottom). The lower and upper ends of the black vertical bars show the "Domain only" and "Domain + Escaped" predictions, respectively, for each model from the upper half of Table 2. For Model A, we plot the value obtained by integrating $M_{\text {ion }}(t)$ up to the end of the simulation (i.e., A from Table 2, rather than $A^{*}$ ). These predicted column densities are lower limits, as we did not follow the high ions for the entire lifetime of each cloud. The gray vertical bars, offset slightly to the right, show the adjusted column density predictions from the lower half of Table 2. These predictions take into account the estimated contribution from ions beyond the ends of the simulations (see Section 4.3 for details). The horizontal solid lines show the observed halo column densities. For C IV we plot the average value of $\log [N(\mathrm{C}$ IV $) \sin |b|]$, with the dashed lines indicating the standard deviation (Savage \& Wakker 2009, Table 4, specifically the values for all extragalactic sightlines). For $\mathrm{N} \mathrm{v}$ we plot the best-fit value of the midplane ion density multiplied by the ion scale height, with the dashed lines indicating the uncertainty (Savage et al. 1997, Table 7, specifically the values that properly take into account the fact that some sightlines yielded only upper limits on the $\mathrm{Nv}$ column density). We assume that the contribution from the Local Bubble is negligible for these ions. For O VI we plot the average value of $\log [N(\mathrm{O} v \mathrm{~V}) \sin |b|]$, with the dashed lines indicating the standard deviation (Savage et al. 2003, Table 3, specifically the values for the full data set). We have subtracted $7 \times 10^{12} \mathrm{~cm}^{-2}$ to allow for the contribution from the Local Bubble (Oegerle et al. 2005). 
Table 2

Average Column Densities Predicted for the Galactic Halo

\begin{tabular}{|c|c|c|c|c|c|c|c|}
\hline \multirow[t]{2}{*}{ Model } & \multirow[t]{2}{*}{$\beta_{\mathrm{HVC}}{ }^{\mathrm{a}}$} & \multicolumn{2}{|c|}{$\begin{array}{c}\bar{N}\left(\mathrm{C}_{\mathrm{IV}}\right) \\
\left(10^{13} \mathrm{~cm}^{-2}\right)\end{array}$} & \multicolumn{2}{|c|}{$\begin{array}{c}\bar{N}(\mathrm{~N} \mathrm{v}) \\
\left(10^{13} \mathrm{~cm}^{-2}\right)\end{array}$} & \multicolumn{2}{|c|}{$\begin{array}{c}\bar{N}(\mathrm{O} \text { vI }) \\
\left(10^{13} \mathrm{~cm}^{-2}\right)\end{array}$} \\
\hline & & $\begin{array}{c}\text { Domain } \\
\text { Only }\end{array}$ & $\begin{array}{c}\text { Domain + } \\
\text { Escaped }\end{array}$ & $\begin{array}{c}\text { Domain } \\
\text { Only }\end{array}$ & $\begin{array}{c}\text { Domain + } \\
\text { Escaped }\end{array}$ & $\begin{array}{c}\text { Domain } \\
\text { Only }\end{array}$ & $\begin{array}{c}\text { Domain + } \\
\text { Escaped }\end{array}$ \\
\hline A & $\ldots$ & 0.27 & 0.31 & 0.13 & 0.27 & 1.1 & 6.1 \\
\hline $\mathrm{A}^{*}$ & $\ldots$ & 0.088 & 0.088 & 0.043 & 0.044 & 0.35 & 0.41 \\
\hline B & $\ldots$ & 0.26 & 0.30 & 0.10 & 0.13 & 0.99 & 1.5 \\
\hline $\mathrm{C}$ & $\ldots$ & 0.14 & 0.18 & 0.043 & 0.072 & 0.32 & 0.96 \\
\hline $\mathrm{D}$ & $\ldots$ & 0.031 & 0.17 & 0.015 & 0.076 & 0.11 & 0.91 \\
\hline $\mathrm{E}$ & $\ldots$ & 0.18 & 0.22 & 0.067 & 0.12 & 0.50 & 1.4 \\
\hline $\mathrm{F}$ & $\ldots$ & 1.1 & 1.4 & 0.34 & 0.44 & 2.7 & 3.8 \\
\hline $\mathrm{G}$ & $\ldots$ & 0.43 & 0.44 & 0.19 & 0.24 & 1.2 & 2.5 \\
\hline \multicolumn{8}{|c|}{ Adjusted column density predictions (see Section 4.3) } \\
\hline A & 0.721 & 0.37 & 0.43 & 0.18 & 0.37 & 1.6 & 8.5 \\
\hline $\mathrm{A}^{*}$ & $0.272^{*}$ & 0.32 & 0.32 & 0.16 & 0.16 & 1.3 & 1.5 \\
\hline $\mathrm{B}$ & 0.216 & 1.2 & 1.4 & 0.48 & 0.60 & 4.6 & 6.8 \\
\hline $\mathrm{C}$ & 0.344 & 0.40 & 0.51 & 0.12 & 0.21 & 0.92 & 2.8 \\
\hline $\mathrm{D}$ & 0.257 & 0.12 & 0.65 & 0.057 & 0.29 & 0.44 & 3.6 \\
\hline $\mathrm{E}$ & 0.228 & 0.78 & 0.97 & 0.29 & 0.54 & 2.2 & 6.1 \\
\hline $\mathrm{F}$ & 0.327 & 3.4 & 4.3 & 1.0 & 1.3 & 8.4 & 12 \\
\hline $\mathrm{G}$ & 0.272 & 1.6 & 1.6 & 0.69 & 0.87 & 4.6 & 9.3 \\
\hline
\end{tabular}

Notes. These column densities were calculated using Equation (3). The column densities in the upper half of the table were calculated by integrating $M_{\text {ion }}(t)$ up to the end of each simulation, except where noted. As a result, these column densities are lower limits, because they neglect the contributions from ions at later times. The adjusted column densities in the lower half of the table are those from the upper half divided by $\beta_{\mathrm{HVC}}$, to take into account the estimated contribution from ions beyond the ends of the simulations (see Section 4.3 for details). For each ion, the first column gives the predicted column density calculated only from the ions in the domain. The second column includes the ions that have escaped from the domain (see Section 3 ).

a The fraction of the HVC's initial H I mass that has ablated and/or ionized by the end of the simulation (except where noted), from Paper I. This quantity was not used in the upper half of the table, so we do not include it there.

* Calculated by integrating $M_{\mathrm{ion}}(t)$ to $t=16 \mathrm{Myr}$, which corresponds to the same stage of evolution as the ends of Models B and F. Similarly, $\beta_{\mathrm{HVC}}$ is taken at $t=16 \mathrm{Myr}$, rather than at the end of the simulation.

\subsection{Model Comparison}

With few exceptions, the Domain-only and Domain + Escaped predictions for each model agree with each other within a factor of $\approx 2$. The exceptions are all three ions in Model D, and $\mathrm{O}$ VI in Models A, C, and E. In these cases, the HVCs shift upward, which allows large numbers of low-velocity high ions to escape from the domain during the course of the simulations.

Comparing models having different velocities (Models B, C, and D), we find that their Domain + Escaped predictions agree within a factor of two, as do the Domain-only and the Domain + Escaped predictions for models having different density profiles (Models $\mathrm{C}$ and $\mathrm{E}$ ). This is understandable, given the similarities in the ion masses between these models (see Section 3.2). The Domain-only and the Domain + Escaped predictions for models having different densities (Models B and G) also agree within a factor of two (note that although $M_{\text {ion }}(t)$ in Equation (3) is lower for Model G, so too is $M_{\mathrm{HVC}, 0}^{\mathrm{HI}}$ ).

Comparing the results from the different-sized model clouds (Models A, B, and F) is a little more complicated, as the simulated portions of the clouds' lifetimes are not equivalent. As noted in Section 3.2, the simulation times must be divided by $r_{0}$ if we wish to compare different-sized HVCs at equivalent stages in their evolution. Hence, we must reset the end time for the $M_{\text {ion }}(t)$ integration, $t_{\mathrm{f}}$, such that $t_{\mathrm{f}} / r_{0}$ is the same for all clouds. We achieve this by using the full simulation times as $t_{\mathrm{f}}$ for Models B and F (120 and $240 \mathrm{Myr}$, respectively), and setting $t_{\mathrm{f}}$ to $16 \mathrm{Myr}$ for Model A. The column density predictions resulting from the new integration of the Model A results are labeled $A^{*}$ in Table 2.
Having obtained column density predictions for equivalent portions of the clouds' lifetimes, we can compare them to the expectations for the simple model of a spherical cloud mentioned in Section 3.2. In this simple model, if $t_{\mathrm{f}} / r_{0}$ is the same for all clouds, we expect $\bar{N}$ (ion) $\propto r_{0}$ (see Equation (A3) in the Appendix). The $r_{0}$ ratios for Models $\mathrm{A}^{*}: \mathrm{B}: \mathrm{F}$ are $0.13: 1: 2$ (Table 1). In comparison, the ratios of the Domain + Escaped column densities are 0.29:1:4.7 for $\mathrm{C}$ IV, 0.34:1:3.4 for $\mathrm{N} \mathrm{v}$, and 0.28:1:2.6 for O VI. The ratios of the Domain-only column densities are generally similar: $0.34: 1: 4.3,0.41: 1: 3.3$, and $0.35: 1: 2.8$ for $\mathrm{C}$ IV, $\mathrm{N} \mathrm{V}$, and $\mathrm{O}$ VI, respectively. Hence, the column densities increase with $r_{0}$, but not linearly. The fact that the column densities for Models $\mathrm{A}^{*}$ and $\mathrm{F}$ are somewhat larger relative to the Model $\mathrm{B}$ values than expected from $r_{0}$ is connected to the observation that the relationship between $M_{\text {ion }}\left(t / r_{0}\right)$ and $M_{\mathrm{HVC}, 0}^{\mathrm{HI}}$ is generally somewhat shallower than linear proportionality between Models $A *$ and $B(\gamma \approx 0.8-1.0)$, and generally somewhat steeper than linear proportionality between Models $\mathrm{B}$ and $\mathrm{F}(\gamma \approx 0.9-1.6$; see Section 3.2).

For Model A, we can extend the integral of $M_{\text {ion }}(t)$ to later times, and naturally $\bar{N}$ (ion) increases as a result. However, we do not have data from Models B and $\mathrm{F}$ for later times with which to compare the Model A results.

\subsection{Including the Contribution from Ions Beyond the Ends of the Simulations}

Here, we describe a method for estimating the contributions to the column densities from ions after the simulations have ended. From Equation (3), we see that the predicted column densities 
depend on the integral $\int M_{\mathrm{ion}}(t) d t$. Ideally, the time integration should be carried out over the entire lifetime of the high ions in the halo, but in Section 4.1 we carried out the integration only up to the end of each simulation at time $t=T_{\text {sim }}$. To estimate the contribution from ions beyond the ends of the simulations, we assume that each and every unit mass of H I "lost" from the HVC to ablation and ionization makes an approximately equal contribution to $\int M_{\text {ion }}(t) d t$. In this case, the integral $\int M_{\text {ion }}(t) d t$ (integrated over all time) in Equation (3) can be replaced by $\int_{0}^{T_{\text {sim }}} M_{\text {ion }}(t) d t /\left(M_{\mathrm{HVC}, \text { lost }}^{\mathrm{HI}} / M_{\mathrm{HVC}, 0}^{\mathrm{HI}}\right) \equiv \int_{0}^{T_{\text {sim }}} M_{\mathrm{ion}}(t) d t / \beta_{\mathrm{HVC}}$, where $M_{\mathrm{HVC} \text { lost }}^{\mathrm{HI}}$ is the mass of $\mathrm{Hi}$ lost from the HVC to ablation and ionization, and $\beta_{\mathrm{HVC}}$ is the ratio of the lost mass to the HVC's initial Hi mass (defined in Paper I). Substituting this new integral into Equation (3) allows us to approximate the column densities that would be predicted if we could follow the full evolution of the cloud. In practice, this substitution can be accomplished by dividing the column densities calculated in Section 4.1 by $\beta_{\mathrm{HVC}}$.

The lower half of Table 2 contains the adjusted column density predictions for Models $\mathrm{A}-\mathrm{G}$, obtained by dividing the values in the upper half of the table by $\beta_{\mathrm{HVC}}$. These adjusted column density predictions are also plotted in Figure 6 , in gray. The adjustment increases the column densities for Models B-G by factors of $\sim 3-5(\sim 0.5-0.7 \mathrm{dex})$, and those for Model A by $\approx 40 \%$ ( $0.14 \mathrm{dex})$. The lower half of Table 2 also contains the adjusted column densities for Model A*, obtained by dividing the relevant values in the upper half of the table by the value of $\beta_{\mathrm{HVC}}$ at $t=16 \mathrm{Myr}$.

After dividing by $\beta_{\mathrm{HVC}}$, the Domain-only column density predictions for Model A are systematically higher than those for Model A*. As the Model A predictions were obtained by integrating the ion masses to a later time than the Model A* predictions, this discrepancy suggests that we are still underestimating the contribution from ions at times later than the ends of the simulations. Nevertheless, the Domain-only predictions for Models $\mathrm{A}$ and $\mathrm{A} *$ agree within $25 \%$, which gives us some confidence that dividing by $\beta_{\mathrm{HVC}}$ leads to reasonable estimates of the contribution of ions from beyond the ends of the simulations to the column densities. It should be noted that the Domain + Escaped predictions for Models $\mathrm{A}$ and $\mathrm{A}^{*}$ in the lower half of Table 2 are less consistent than the Domainonly predictions, especially for $\mathrm{NV}$ and $\mathrm{O}$ VI. However, the Domain + Escaped predictions for Model A may be significantly overestimated, as relatively large quantities of low-velocity $\mathrm{N} \mathrm{V}$ and $\mathrm{O}$ vI were swept off the top of the domain in this simulation, and we were unable to follow these ions' subsequent evolution. Hence, the discrepancy in the adjusted Domain + Escaped predictions for Models $\mathrm{A}$ and $\mathrm{A} *$ does not argue against the reliability of our method for estimating the contribution from ions beyond the ends of the simulations.

It should be noted that there is a time delay between a unit mass of cool material being stripped from the cloud and that material becoming rich in high ions. As a result, the abovedescribed division by $\beta_{\mathrm{HVC}}$ will neglect the contribution to $\int M_{\text {ion }}(t) d t$ from material that has been stripped from the cloud, but has not yet completed its ionization evolution by the end of the simulation. However, as this neglected contribution to $\int M_{\text {ion }}(t) d t$ is likely to be small, we do not attempt to further adjust our column density predictions to take it into account.

\subsection{Comparison with Observations}

Owing to the large number of models examined and the multiple methods for estimating their contributions to the column densities of low-velocity high ions, our column density predictions cover a wide range. At the high end, they can account for $\gtrsim 1 / 3$ of the observed O VI column density, while our lower limits account for only a few percent of the observed C IV.

Let us first consider the column densities from the upper half of Table 2 (the values that exclude the contributions from the times beyond the ends of the simulations). These are plotted in black in Figure 6, where they are compared with the observed values $\left(10 \times 10^{13}, 2.5 \times 10^{13}\right.$, and $15.5 \times 10^{13} \mathrm{~cm}^{-2}$ for $\mathrm{C} \mathrm{IV}$, Nv, and O vi, respectively; Savage \& Wakker 2009; Savage et al. 1997, 2003; see figure caption for details). In general, we find that these model column densities account for only a small fraction of the low-velocity $\mathrm{CIV}, \mathrm{N} \mathrm{v}$, and $\mathrm{O}$ vi observed in the halo. The exceptions are Models A and F, whose Domain + Escaped $\mathrm{O}$ VI predictions agree with the observed value within a factor of $\sim 2.5$ and $\sim 4$, respectively.

When we include the estimated contribution from the times beyond the ends of the simulations (tabulated in the lower half of Table 2, plotted in gray in Figure 6), we see that several models' predictions come within a factor of $\sim 3$ of the observed $\mathrm{O}$ vi column density. The Domain-only and Domain + Escaped predictions from Models B and $G$ are within a factor of 3.5 of the observed value, while the Domain-only and Domain + Escaped predictions from Model F and the Domain + Escaped prediction from Model A are within a factor of two of the observed value. The Domain-only predictions are of particular interest, as they are lower limits on the true model predictions. This is because the original Domain-only predictions are strict lower limits on the true predictions (see Section 4.1), and dividing by $\beta_{\mathrm{HVC}}$ may underestimate the contribution from times beyond the ends of the simulations (see Section 4.3). Hence, our HVC models can account for a significant fraction of the low-velocity $\mathrm{O}$ VI observed in the halo (e.g., our reference model, Model B, can account for $30 \%-44 \%$ of the observed O VI, where the lower limit is taken from the Domain-only prediction and the upper limit is taken from the Domain + Escaped prediction).

For C IV and Nv, we find that fewer of our models predict column densities that are within a factor of $\sim 3$ of the observed values. The Domain + Escaped C IV and N v predictions from Model $\mathrm{F}$ that include the contributions from times beyond the end of the simulation agree with the observed values within factors of three and two, respectively, while the equivalent Model G N v prediction agrees with the observed value within a factor of three. The other models typically account for $\lesssim 15 \%$ and $\lesssim 25 \%$ of the observed $\mathrm{C}$ IV and $\mathrm{Nv}$, respectively (e.g., our reference model, Model B, can account for only 12\%-14\% of the observed CIV, where the lower and upper limits are again taken from the Domain-only and Domain + Escaped predictions, respectively). We will discuss these results further in Section 6.

\section{COLUMN DENSITY PROFILES}

In this section, we present $\mathrm{O}$ vi column density profiles (i.e., column density as a function of velocity) derived from one of our HVC models and compare them in general terms with the observed profiles. Note that, although the main topic of this paper is low-velocity high ions, these column density profiles span both low and high velocities.

Figure 7 shows OVI profiles for four different vertical sightlines from two epochs of Model B, and Figure 8 shows profiles for five different horizontal sightlines from one epoch of the same model. The column density of each hydrodynamical cell along the line of sight was calculated from the gas density 

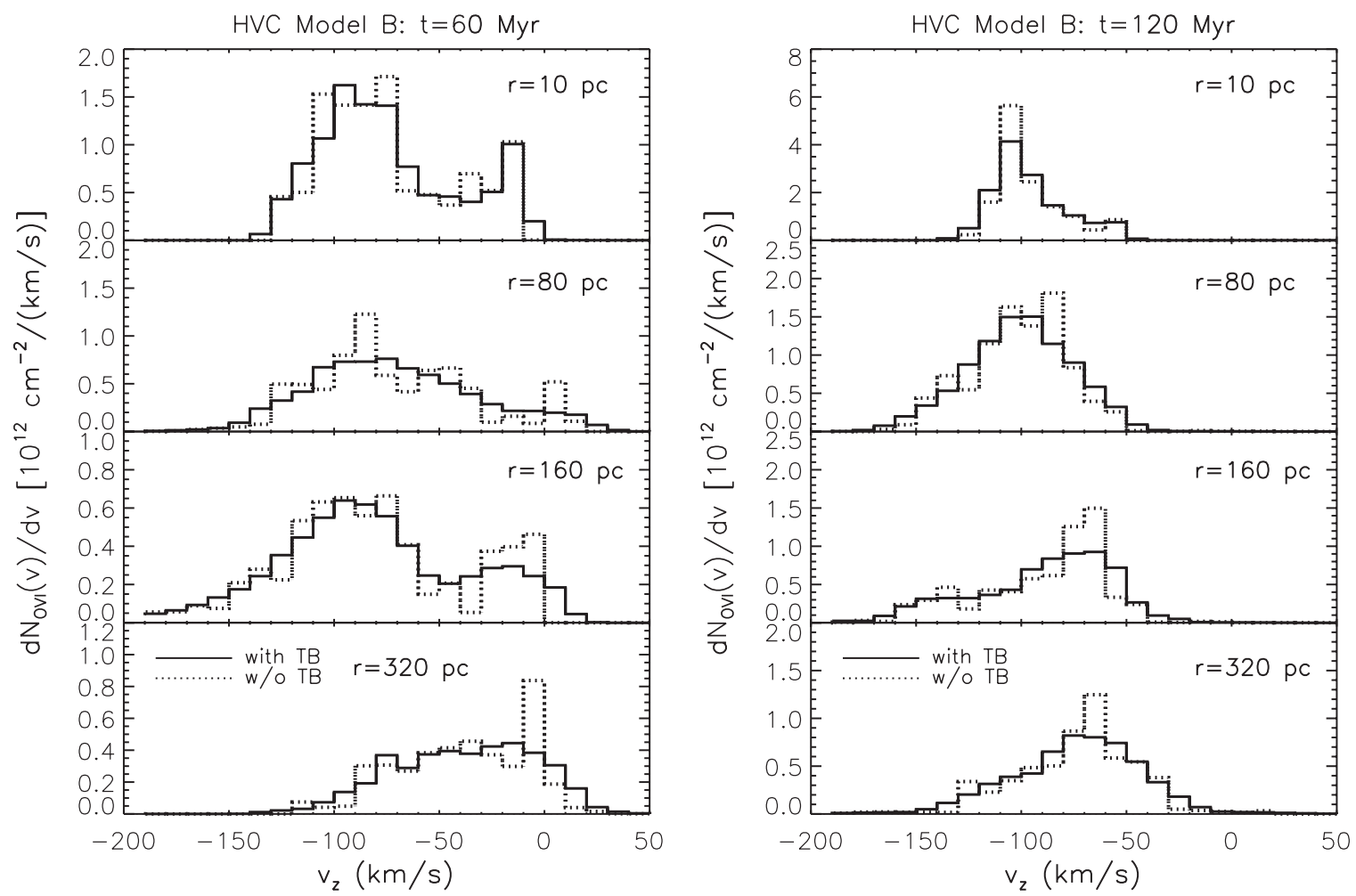

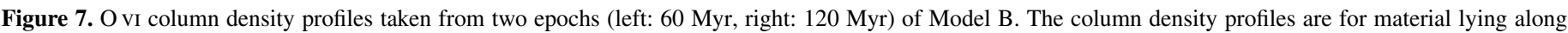

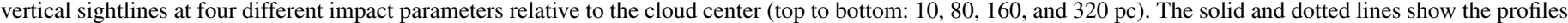
with and without thermal broadening (see the text for details).

and the relevant ion fraction in that cell; the velocity bin was chosen according to the cell's line-of-sight velocity, $v$, in the observer's frame (for the vertical and horizontal sightlines, the observer is located below and to the right of the model domain, respectively). The dotted column density profiles in Figures 7 and 8 do not include thermal broadening and were constructed by summing the contributions from all the cells along the line of sight, assuming that each cell's column density profile is a Dirac $\delta$ function at $v$. The solid column density profiles do include thermal broadening, which we approximated by convolving each cell's column density profile with a boxcar of full width $2 b$, where $b=\left(0.129 \mathrm{~km} \mathrm{~s}^{-1}\right) \sqrt{(T / \mathrm{K}) / A}$ is the thermal velocityspread parameter, $T$ is the gas temperature in the cell, and $A$ is the relative atomic mass of the ion in question (Spitzer 1978, Equation (3-21)). Note that the profiles in Figures 7 and 8 are much broader than the FUSE spectral resolution $\left(\sim 20 \mathrm{~km} \mathrm{~s}^{-1}\right.$; Sahnow et al. 2000; Wood et al. 2002).

Figures 7 and 8 show that the column density profiles with and without thermal broadening are similar to each other, regardless of epoch or sightline. This means that the broadening of the profiles is due to variation in the bulk fluid velocity along the line of sight, rather than thermal broadening. For the horizontal sightlines, this variation in the bulk fluid velocity is due to turbulence in the mixed gas. For the vertical sightlines, however, there is an additional effect. The ions' velocities tend toward that of the ambient medium $(v=0$ in the observer's frame) the further they are from the HVC. Therefore, a vertical sightline through our model domains will typically sample both high- and low-velocity ions, spanning a continuous range of velocities. As a result of this additional effect, the column density profiles for the vertical sightlines are generally somewhat broader than those for the horizontal sightlines.
FUSE observations of $\mathrm{O}$ VI absorption in the halo show some qualitative agreement and some qualitative disagreement with our predicted column density profiles. The observed line widths are generally much broader than the expected thermal width, as in our model profiles. The observed velocity-spread parameters for low- and high-velocity $\mathrm{O}$ VI absorption are typically $b \sim 50-70$ and $\sim 30-50 \mathrm{~km} \mathrm{~s}^{-1}$, respectively (Savage et al. 2003; Sembach et al. 2003), compared with $b=18 \mathrm{~km} \mathrm{~s}^{-1}$ for thermal broadening at $T=3 \times 10^{5} \mathrm{~K}$. For several sightlines, absorption is observed over a continuous range of velocities from $v \sim 0$ to $v \sim-200$ or $+200 \mathrm{~km} \mathrm{~s}^{-1}$ (see Figure 1 in Wakker et al. 2003), in qualitative agreement with our model column density profiles for vertical sightlines. However, the observed column density profiles typically have relatively more ions near $v=0$ than our model profiles. These extra ions near $v=0$ are likely due to other sources of low-velocity high ions, in addition to those predicted by our HVC models. We discuss further the idea of multiple sources of high ions in the halo in Section 6.2.

\section{DISCUSSION}

We have analyzed existing simulations of HVCs (Paper I) in order to estimate the quantities of low-velocity ions that result from the passage of HVCs through the hot halo. In Section 4.4, we showed that some of our models could account for a significant fraction of the observed low-velocity $\mathrm{O}$ VI, but not of the low-velocity $\mathrm{C}$ IV or $\mathrm{N}$ v. In this section, we first discuss our model assumptions, in particular neglected physical processes (Section 6.1.1), the pressure and temperature of the halo (Section 6.1.2), and the factors in Equation (3) for which we had to assume values (Section 6.1.3). Then, in Section 6.2, we discuss our model alongside other models of the high ions in the halo. 


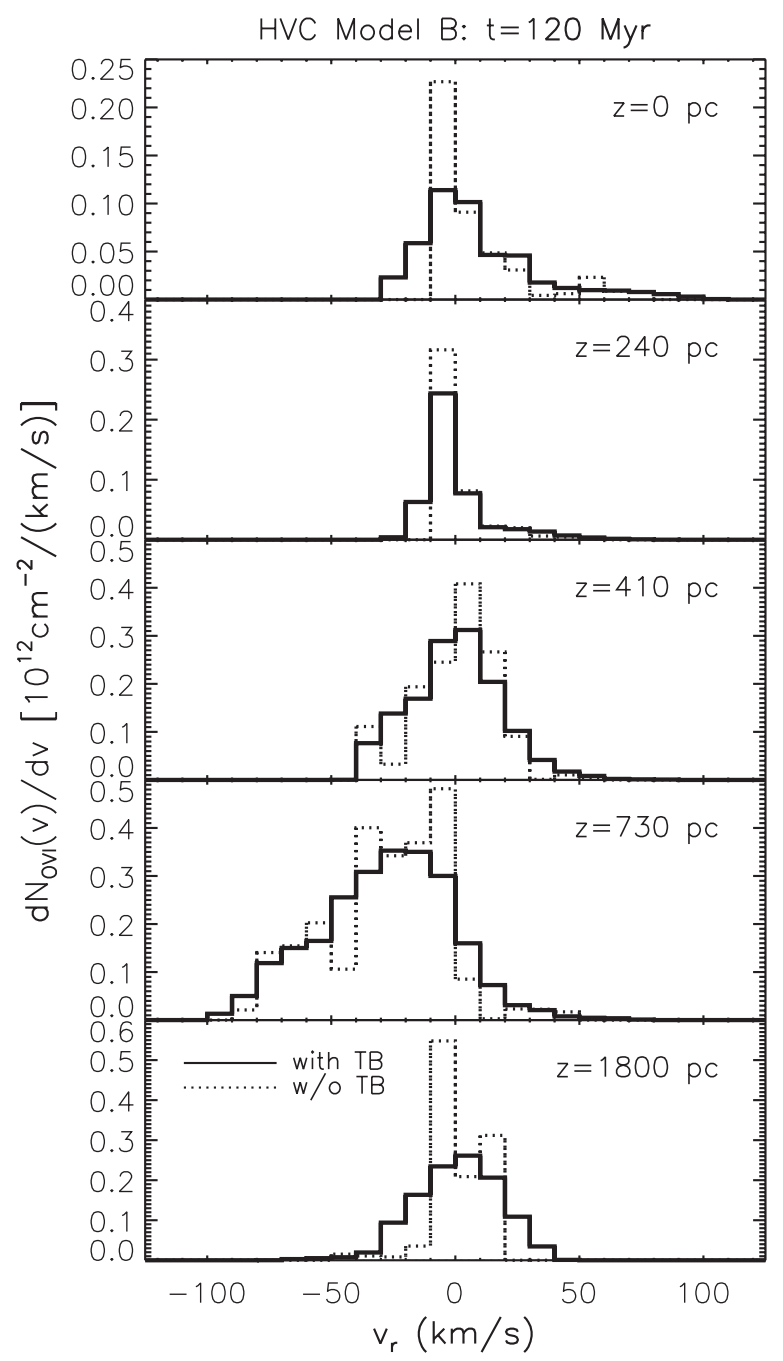

Figure 8. As Figure 7, but for horizontal sightlines through the Model B domain at $t=120$ Myr. Profiles are plotted for sightlines at five different heights, measured relative to the cloud's initial position in the domain (top to bottom: 0 , 240, 410, 730, and $1800 \mathrm{pc})$.

\subsection{Model Assumptions}

\subsubsection{Neglected Physical Processes}

Our model does not include a magnetic field, as our twodimensional geometry prevents our modeling realistic field configurations. Magnetic fields are known to suppress the development of turbulence (e.g., Ryu et al. 2000), while the development of turbulence differs in two and three dimensions (e.g., Stone \& Norman 1992). It is unclear whether adding a magnetic field and a third dimension to the model would result in more or fewer high ions, compared to our current simulations. Kwak \& Shelton (2010) point out that their two-dimensional hydrodynamic simulations of plane-parallel mixing layers predict ion column density ratios that are similar to those predicted by the three-dimensional magnetohydrodynamic simulations of Esquivel et al. (2006). However, we are unable to make a similar statement regarding the magnitudes of the column densities.

Our model also does not include thermal conduction. Like a magnetic field, thermal conduction may suppress the growth of instabilities (Orlando et al. 2008), although we cannot predict the extent to which thermal conduction would affect the development of turbulence in our simulations. Assuming that turbulence is able to develop, the inclusion of thermal conduction in our simulations would be unlikely to greatly affect our results, as turbulent diffusion of heat should dominate over diffusion by thermal conduction (see Section 5 of Paper I).

\subsubsection{The Pressure and Temperature of the Halo}

Our model assumes that the halo pressure is uniform over the distance that the cloud falls through the halo ( $\gtrsim 10 \mathrm{kpc}$, assuming a cloud speed of $100 \mathrm{~km} \mathrm{~s}^{-1}$ and a lifetime of $\gtrsim 100 \mathrm{Myr}$ ). This would be an unrealistic assumption for HVCs traveling through the lower halo, where the total interstellar pressure more than doubles from 3600 to $8700 \mathrm{~cm}^{-3} \mathrm{~K}$ between $|z|=3$ and $1 \mathrm{kpc}$ (Ferrière 1998). However, the ambient halo pressure in our model is much lower than this $\left(230 \mathrm{~cm}^{-3} \mathrm{~K}\right.$ in most models, $23 \mathrm{~cm}^{-3} \mathrm{~K}$ in Model $\mathrm{G}$ ), and may be more appropriate for HVCs traveling through the upper halo $(|z| \gtrsim 10 \mathrm{kpc})$. Although the pressure in the upper halo is uncertain, in Paper I we noted that our chosen ambient density $\left(n_{\mathrm{H}}=1.0 \times 10^{-4} \mathrm{~cm}^{-3}\right.$ for Models A-F) is similar to some previous observational estimates of the density in the upper halo $\left(\sim(1-\mathrm{few}) \times 10^{-4} \mathrm{~cm}^{-3}\right.$ for $|z| \gtrsim 10$ kpc; e.g., Weiner \& Williams 1996; Peek et al. 2007; Grcevich \& Putman 2009). As these observational estimates of the density in the upper halo vary by only a factor of a few over a wide range of heights above the disk (tens of $\mathrm{kpc}$ ), the pressure gradients will not be large in the upper halo if the temperature is reasonably uniform in this region.

The origin of HVCs remains uncertain, although Peek et al. (2007) point out that the observation of $\mathrm{HVCs} \gtrsim 10 \mathrm{kpc}$ above the disk argues against their origin in a Galactic fountain (Bregman 1980). Some HVCs have low metallicities (e.g., $\sim 0.13$ solar for Complex C; Collins et al. 2007), which also argues against their being composed primarily of fountain material. If the majority of HVCs originate in extragalactic or circumgalactic material, they would travel great distances through the upper halo, in a relatively low pressure, low density environment similar to that in our models. As noted above, the pressure gradients may not be large in the upper halo. Furthermore, clouds that have transverse components to their motion through the halo would experience less change in ambient pressure in a vertically stratified halo than if they were traveling vertically downward. Modeling the change in ambient pressure as an HVC falls into the Galaxy is beyond our current models, but could be incorporated into future simulations.

Our model also assumes a halo temperature of $1 \times 10^{6} \mathrm{~K}$. $\mathrm{X}$-ray observations indicate the presence of $\sim$ million-degree gas in the halo, although its temperature structure and filling factor remain uncertain. We did not investigate different ambient temperatures in our suite of models. However, in their simulations of two-dimensional plane-parallel mixing layers, Kwak \& Shelton (2010) found that increasing the temperature on the hot side of the interface from $1 \times 10^{6}$ to $3 \times 10^{6} \mathrm{~K}$ did not have a large effect on the ion column densities for sightlines perpendicular to the interface.

Kwak \& Shelton (2010) did not carry out a corresponding simulation with a temperature lower than $1 \times 10^{6} \mathrm{~K}$. However, if the halo temperature were much lower than $1 \times 10^{6} \mathrm{~K}$, the halo's $R O S A T R 2 / R 1$ ratio would be lower than the observed value (e.g., $R 2 / R 1=0.48$ for a $5 \times 10^{5} \mathrm{~K}$ plasma, while observational analyses yield $R 2 / R 1 \gtrsim 0.5$ for the halo's intrinsic emission; Snowden et al. 2000; Kuntz \& Snowden 2000). If the ambient temperature were even lower, then the ambient medium would not persist in the upper halo, as it would have a relatively short cooling time. For example, for an ambient medium with $T=3 \times 10^{5} \mathrm{~K}$ and $n_{\mathrm{H}}=1.0 \times 10^{-4} \mathrm{~cm}^{-3}$, the cooling time 
would be $\sim 100 \mathrm{Myr}$ (calculated using the 1993 version of the Raymond \& Smith 1977 code). This time is similar to the lengths of our simulations.

$$
\text { 6.1.3. } \dot{\mathcal{M}}_{H V C}^{H}, R_{M W} \text {, and Elemental Abundances }
$$

When we used Equation (3) to calculate the column density of a given ion from our hydrodynamical simulations, we had to assume values for three important factors: $\dot{\mathcal{M}}_{\mathrm{HVC}}^{\mathrm{H}}, R_{\mathrm{MW}}$, and the elemental abundance (the abundance affects the values of $M_{\text {ion }}(t)$ derived from our simulations). As noted in Section 4.1, the value of $\dot{\mathcal{M}}_{\mathrm{HVC}}^{\mathrm{H}}$ is uncertain. However, our assumed value of $0.5 M_{\odot} \mathrm{yr}^{-1}$ should be reasonably conservative (in the sense of tending to lead to underestimates of the predicted column densities), given the large infall rates associated with individual complexes (e.g., Complex C, the Smith Cloud, and the Magellanic Stream). The radius $R_{\mathrm{MW}}$ over which the halo ions are distributed is more uncertain. By choosing $R_{\mathrm{MW}}=25 \mathrm{kpc}$, we have assumed the ions that result from HVCs interacting with the hot halo are spread uniformly above the entire stellar disk. However, if these ions tend to be concentrated toward the center of the Galaxy, which is a reasonable assumption, then our choice of $R_{\mathrm{MW}}$ should also be reasonably conservative.

For the elemental abundances, we used the interstellar values from Wilms et al. (2000), which are in good agreement with recent measurements of solar photospheric abundances (Lodders 2003; Asplund et al. 2009). ${ }^{6}$ The Wilms et al. (2000) interstellar abundances of carbon and oxygen include atoms that are in dust: the gas-phase abundances of carbon and oxygen in the local ISM are 0.23 and 0.19 dex lower than the Wilms et al. values, respectively (Cardelli et al. 1996; Meyer et al. 1998). Nitrogen, however, is not depleted onto dust (Meyer et al. 1997). Carbon, nitrogen, and oxygen abundances in the halo are more uncertain, but in the lower halo at least $(|z| \lesssim 2 \mathrm{kpc})$, total abundances appear to be approximately solar (based on the gas-phase sulfur abundance), and there is less depletion onto dust than in the disk for elements heavier than oxygen (Savage \& Sembach 1996). If the carbon, nitrogen, and oxygen abundances are also approximately solar in the halo, and less depleted onto dust than in the disk, then our $M_{\text {ion }}(t)$ predictions for C IV and O VI would need to be revised downward by no more than $\sim 40 \%$ to compensate for the possibility that these elements are depleted onto dust, while our $\mathrm{N} v$ predictions would not need revision.

However, it should be noted that varying the abundances would affect the cooling curve used in our simulations. While the FLASH manual is not explicit, the FLASH cooling curve is likely based on a cooling curve calculated with Allen (1973) abundances, as these are the defaults for the Raymond \& Smith (1977) code. Modifying the cooling curve would affect the quantities of high ions predicted by our simulations, by affecting the amount of gas at optimal temperatures for such ions. Quantifying the extent of this effect is beyond the scope of this paper. However, while lowering the abundances would reduce $M_{\text {ion }}(t)$, it would also reduce the cooling rate, meaning that gas would remain at high-ion-rich temperatures for longer. As a result, lowering the abundances would not necessarily lead to a commensurate reduction in $\int M_{\text {ion }}(t) d t$, and hence in the predicted column densities.

\footnotetext{
6 Note that the ion masses and column densities in Paper I were calculated using Allen (1973) abundances, which are 0.14, 0.08, and 0.13 dex larger than the Wilms et al. (2000) abundances for carbon, nitrogen, and oxygen, respectively.
}

\subsection{A Composite Model of the High Ions in the Galactic Halo}

We pointed out in Section 4.4 that the Domain-only column density predictions in the lower half of Table 2 are lower limits on the true model predictions. In the previous subsection, we argued that our choices of $\dot{\mathcal{M}}_{\mathrm{HVC}}^{\mathrm{H}}$ and $R_{\mathrm{MW}}$ should be reasonably conservative, and that adjusting the elemental abundances is unlikely to result in large adjustments in the predicted column densities. Hence, our statement in Section 4.4 that our HVC models can account for a significant fraction of the low-velocity $\mathrm{O}$ vi observed in the halo is quite robust.

While the Domain-only predictions from the lower half of Table 2 are lower limits, the corresponding Domain + Escaped predictions are generally upper limits on the expected ion column densities. This is because, for most models, the numbers of low-velocity ions in the material that has flowed off the domain decrease with time (see Section 3). Therefore, taking these lower and upper limits for Model B (our reference model), we find that HVCs could account for $30 \%-44 \%$ of the lowvelocity $\mathrm{O}$ VI in the halo, but only $12 \%-14 \%$ of the low-velocity C IV (Section 4.4).

The preceding statement raises the question: why can our model account for a significant fraction of the $\mathrm{O}$ VI in the halo, but not of the C IV? The answer is likely because our model does not include photoionization, which can increase the amount of C IV relative to $\mathrm{O}$ VI, due to C IV's lower ionization potential. For example, the cooling Galactic fountain model of Shapiro \& Benjamin (1993), which includes photoionization, predicts $\sim 2-7$ times as much $\mathrm{C}$ IV relative to $\mathrm{O}$ VI as the cooling fountain model of Edgar \& Chevalier (1986), which does not. Models that include photoionization (e.g., Ito \& Ikeuchi 1988) can explain the enhancement in C IV (and Si IV, which is not studied in this paper) relative to $\mathrm{N} v$ observed at large $|z|$ (Savage et al. 1997). However, it should be noted that the Ito \& Ikeuchi (1988) model also predicts that $\mathrm{C}$ IV should be enhanced relative to $\mathrm{O}$ VI at large $|z|$, which appears not to be the case observationally (Savage et al. 2003).

As our HVC model does not include photoionization, and therefore tends to significantly underpredict the amount of C IV in the halo, we are not putting it forward as the only source of high ions in the halo and as an alternative to other models. Instead, we are pointing out that a complete model of the Galactic halo should include the contribution of HVCs to the low-velocity high ions, particularly O VI. While developing a complete self-consistent model of the high ions in the halo is beyond the scope of this paper, we will give an example of how different existing models of the halo high ions could be pieced together. Note that we consider only the total column densities of the ions, not their $z$ distributions, as we cannot derive the $z$ distributions of the ions from our current HVC model. However, if the HVCs spend most of their lifetimes in the upper halo (see Section 6.1.2), then the interactions of the HVCs with the ambient gas may result in a large number of high ions at large distances from the plane.

Our example composite model includes contributions from HVCs (this paper, specifically our reference model, Model B), extraplanar SNRs (Shelton 2006), radiatively cooling Galactic fountains (Shapiro \& Benjamin 1993), and photoionization by an external radiation field (Ito \& Ikeuchi 1988). The contributions from these various components to the observed column densities are presented in Table 3 (see the footnotes to that table for more details). Note that the overall normalization 
Table 3

Composite Model of Low-velocity High Ions in the Halo

\begin{tabular}{|c|c|c|c|}
\hline & CIV & $\mathrm{N} \mathrm{V}$ & O VI \\
\hline Observed $^{\mathrm{a}}\left(10^{13} \mathrm{~cm}^{-2}\right)$ & 10 & 2.5 & 15.5 \\
\hline (1) $\mathrm{HVCs}^{\mathrm{b}}$ & $13 \%$ & $22 \%$ & $37 \%$ \\
\hline (2) Extraplanar SNRs ${ }^{c}$ & $6 \%$ & $12 \%$ & $17 \%$ \\
\hline (3) Galactic fountains ${ }^{\mathrm{d}}$ & $23 \%-37 \%$ & $14 \%-21 \%$ & $46 \%$ \\
\hline \multicolumn{4}{|l|}{ (4) Photoionization by } \\
\hline external radiation field ${ }^{\mathrm{e}}$ & $39 \%$ & $\ldots$ & $\ldots$ \\
\hline Total & $81 \%-95 \%$ & $47 \%-55 \%$ & $100 \%{ }^{f}$ \\
\hline
\end{tabular}

Notes. Each model component's contribution is expressed as a percentage of the observed column density, in the first row. Any apparent discrepancies between the individual components' contributions and the totals are due to rounding.

a C Iv: Savage \& Wakker (2009); N v: Savage et al. (1997); O vi: Savage et al. (2003), after removing contribution from the Local Bubble (Oegerle et al. 2005).

b Predictions from Model B (our reference model); specifically, the average of the Domain-only and Domain + Escaped predictions from the lower half of Table 2 .

c Shelton (2006), Table 8. We have chosen the model with the median OVI prediction (case 1, drag coefficient 1).

d Shapiro \& Benjamin (1993), Table 1, rescaled such that this component accounts for all of the O VI not already accounted for by HVCs and SNRs. This model includes the effects of self-photoionization.

e Ito \& Ikeuchi (1988), Figure 3(a). We assume that all of the C IV in this figure is due to the photoionized component of their model, and that this component produces negligible $\mathrm{N} \mathrm{V}$ and $\mathrm{O}$ VI.

${ }^{\mathrm{f}}$ Our composite model reproduces $100 \%$ of the O vi by design.

of the Galactic fountain component (component 3 in Table 3) is essentially a free parameter, as the mass flow rate in the fountain is not well known. We therefore rescaled the predictions from Shapiro \& Benjamin (1993) so that this component would account for all of the O VI not accounted for by HVCs and SNRs (this rescaling amounted to multiplying their predictions by $0.24-0.38$, depending on the particular model in their Table 1). Hence, our model accounts for all of the observed $\mathrm{O}$ VI by design.

In our composite model, HVCs, SNRs, and Galactic fountains (components 1-3) can account for approximately half of the observed C IV and N v. The Galactic fountain model of Shapiro \& Benjamin (1993) includes the effects of photoionization by the cooling gas's own radiation, but not by an external radiation field. Photoionization by the extragalactic background, or by radiation from hot stars escaping from the disk, may be able to account for the shortfall in C IV. For example, the hybrid model of Ito \& Ikeuchi (1988) includes a photoionized component with $T=10^{4.0} \mathrm{~K}$, which could account for $\approx 40 \%$ of the observed C IV (component 4 in Table 3), i.e., most or nearly all of the observed C IV is now accounted for. However, this photoionized component is unlikely to be able to account for the additional $\mathrm{N} \mathrm{v}$, due to $\mathrm{N} v$ 's higher ionization potential.

The shortfall in $\mathrm{Nv}$ in our composite model is due to the fact that the various components of our composite model all underpredict the $\mathrm{Nv}$ column density relative to the $\mathrm{O}$ VI column density. The predicted ion ratios for HVCs (this paper), extraplanar SNRs (Shelton 2006), and Galactic fountains (Edgar \& Chevalier 1986; Shapiro \& Benjamin 1993) are all in the range $\log [N(\mathrm{~N} \mathrm{v}) / N(\mathrm{O} \mathrm{vI})] \approx-1.3$ to -1.0 . In comparison, the observed value is $\log [N(\mathrm{Nv}) / N(\mathrm{O}$ vI $)]=-0.8($ see Table 3$)$. Some additional source of gas with $T \approx 2 \times 10^{5} \mathrm{~K}$ would be needed to increase the $\mathrm{Nv}$ column density relative to the $\mathrm{C}$ IV and $\mathrm{O}$ vi values, although what this source could be is not obvious. Plane-parallel turbulent mixing layer models tend to give higher $\mathrm{N}$ v-to-O vi ratios $(\log [N(\mathrm{~N} \mathrm{v}) / N(\mathrm{O}$ vI $)]=-0.9$ to -0.4; Slavin et al. 1993; Kwak \& Shelton 2010), but as the composite model already includes mixing between HVC and ambient gas, it is not clear how more turbulent mixing layers could be added to the model.

\section{SUMMARY}

We have presented further results from our NEI hydrodynamical simulations of HVCs (Paper I). In this paper, we concentrated on the low-velocity high ions that result when an HVC passes through the hot halo. These high ions arise from the mixing of cool cloud gas with hot ambient gas. Initially, this mixed high-ion-rich gas is located near the HVC and travels with HVClike velocities, but later it falls behind the HVC and slows to ISM-like velocities, while retaining its high ion content.

We examined a suite of seven models, covering different cloud velocities, cloud densities, cloud density profiles, and cloud sizes. In general, the cloud velocity and density profile have little effect on the masses of the high ions that result. Larger or denser clouds result in greater masses of high ions, as one would qualitatively expect. An important result is that, except for the fastest model HVC $\left(|v|=300 \mathrm{~km} \mathrm{~s}^{-1}\right)$, the quantities of low-velocity ions are generally larger than the quantities of high-velocity ions. This result suggests that HVCs could be an important source of low-velocity high ions in the halo.

We examined this suggestion more quantitatively, using the HVC infall rate to estimate the average column densities of lowvelocity high ions in the halo due to HVCs. After accounting for the contribution from ions at times beyond the ends of our simulations, and being conservative regarding the HVC infall rate, we find that our models can account for $\gtrsim 30 \%$ of the $\mathrm{O}$ vi column density observed in the halo. This implies that the collisionally ionized gas in material shed by HVCs is a significant source of the low-velocity $\mathrm{O}$ vi observed in the halo. In contrast, such gas is probably not a significant source of low-velocity Civ: our reference model can account for only $12 \%-14 \%$ of the observed column density. This is probably because the observed C IV is likely affected by photoionization, which our model does not include.

We used the predictions of our HVC model in a simple composite model of the low-velocity high ions in the halo, in which we combined the contributions from HVCs, extraplanar SNRs, radiatively cooling fountain gas, and photoionization from an external radiation field. By design, this model accounted for all of the observed O vi. We found that the model could account for most or all of the observed C IV, but only about half of the observed $\mathrm{Nv}$. It is not obvious what the source of the additional $\mathrm{N} v$ could be. Although this composite model was constructed in a relatively simple way and failed to account for all the observed ions, we emphasize the point that any complete model of the high ions in the halo should include the contributions from HVCs, particularly to the column density of low-velocity $\mathrm{O}$ vI.

The software used in this work was in part developed by the DOE-supported ASC/Alliance Center for Astrophysical Thermonuclear Flashes at the University of Chicago. The simulations were performed at the Research Computing Center (RCC) of the University of Georgia. We acknowledge use of the R software package ( $\mathrm{R}$ Development Core Team 2008). This work was supported by NASA grant NNX09AD13G, awarded through the Astrophysics Theory and Fundamental Physics Program. D.B.H. acknowledges funding from NASA 
grant NNX08AJ47G, awarded through the Astrophysics Data Analysis Program.

\section{APPENDIX}

\section{A SIMPLE ANALYTICAL MODEL OF A SPHERICAL HVC}

Here, we describe a simple analytical model of a spherical HVC that loses mass at a rate proportional to its surface area (introduced in Section 3.3.3 of Paper I). This model is helpful for comparing the results from hydrodynamical simulations of different-sized HVCs (see Sections 3.2 and 4.2).

We assume that the mass of low-velocity high ions of a given type, $M_{\text {ion }}$, increases with time at a rate that is proportional to the rate at which the HVC sheds its mass, and thus to the HVC's surface area, i.e., $d M_{\text {ion }} / d t=\alpha r^{2}(t)$. In this simple model, the cloud radius decreases linearly with time from its initial value, $r_{0}$ (see Paper I), and so we can rewrite the mass loss rate as $d M_{\text {ion }} / d t=\alpha\left(r_{0}-k t\right)^{2}$. Note that the constants $\alpha$ and $k$ are unimportant, as long as they are independent of $r_{0}$. Note also that we are ignoring the fact that the ions of interest will subsequently recombine or ionize (i.e., there should be an additional, negative term on the right-hand side of the expression for $d M_{\text {ion }} / d t$ ).

We integrate $d M_{\text {ion }} / d t$ with respect to $t$, with the boundary condition $M_{\text {ion }}=0$ at $t=0$. In order to compare differentsized clouds at equivalent stages of their evolution, we express the solution as a function of the rescaled time, $t / r_{0}$, obtaining

$$
M_{\mathrm{ion}}\left(t / r_{0}\right)=\frac{\alpha r_{0}^{3}}{3 k}\left[1-\left\{1-k\left(\frac{t}{r_{0}}\right)\right\}^{3}\right] \text {. }
$$

Hence, we expect that

$$
M_{\text {ion }}\left(t / r_{0}\right) \propto r_{0}^{3} \propto M_{\mathrm{HVC}, 0} .
$$

We use this result in Section 3.2. Note that this result is only valid if $t<r_{0} / k$ and if the rate at which the ions of interest subsequently recombine or ionize is negligible.

We can also use this model to compare the ion column densities expected from different-sized clouds. If the end time, $t_{\mathrm{f}}$, for the integral in Equation (3) is chosen such that $t_{\mathrm{f}} / r_{0}$ is the same for different-sized clouds, then we expect that

$$
\begin{aligned}
\bar{N}(\text { ion }) & \propto \frac{\int_{0}^{t_{\mathrm{f}}} M_{\mathrm{ion}} d t}{M_{\mathrm{HVC}, 0}^{\mathrm{HI}}} \\
& =r_{0} \frac{\int_{0}^{t_{\mathrm{f}} / r_{0}} M_{\mathrm{ion}}\left(t / r_{0}\right) d\left(t / r_{0}\right)}{M_{\mathrm{HVC}, 0}^{\mathrm{HI}}}, \\
& \propto r_{0},
\end{aligned}
$$

where, for the final step, we have used the fact that $M_{\text {ion }}\left(t / r_{0}\right) \propto$ $M_{\mathrm{HVC}, 0}^{\mathrm{HI}}$ in this simple model (Equation (A2)). We use this result in Section 4.2. Again, note that this result is only valid if the rate at which the ions of interest ionize or recombine is negligible.

\section{REFERENCES}

Allen, C. W. 1973, Astrophysical Quantities (3rd ed.; London: Athlone) Asplund, M., Grevesse, N., Sauval, A. J., \& Scott, P. 2009, ARA\&A, 47, 481 Begelman, M. C., \& Fabian, A. C. 1990, MNRAS, 244, 26P

Benjamin, R. A., \& Shapiro, P. R. 1993, in UV and X-Ray Spectroscopy of Astrophysical and Laboratory Plasmas, ed. E. H. Silver \& S. M. Kahn (Cambridge: Cambridge Univ. Press), 280
Blitz, L., Spergel, D. N., Teuben, P. J., Hartmann, D., \& Burton, W. B. 1999, ApJ, 514,818

Böhringer, H., \& Hartquist, T. W. 1987, MNRAS, 228, 915

Borkowski, K. J., Balbus, S. A., \& Fristrom, C. C. 1990, ApJ, 355, 501

Bowen, D. V., Jenkins, E. B., Tripp, T. M., et al. 2008, ApJS, 176, 59

Bregman, J. N. 1980, ApJ, 236, 577

Bregman, J. N., \& Lloyd-Davies, E. J. 2007, ApJ, 669, 990

Brüns, C., Kerp, J., Staveley-Smith, L., et al. 2005, A\&A, 432, 45

Burrows, D. N., \& Mendenhall, J. A. 1991, Nature, 351, 629

Cardelli, J. A., Meyer, D. M., Jura, M., \& Savage, B. D. 1996, ApJ, 467, 334

Collins, J. A., Shull, J. M., \& Giroux, M. L. 2004, ApJ, 605, 216

Collins, J. A., Shull, J. M., \& Giroux, M. L. 2007, ApJ, 657, 271

Dixon, W. V., \& Sankrit, R. 2008, ApJ, 686, 1162

Dixon, W. V., Sankrit, R., \& Otte, B. 2006, ApJ, 647, 328

Edgar, R. J., \& Chevalier, R. A. 1986, ApJ, 310, L27

Esquivel, A., Benjamin, R. A., Lazarian, A., Cho, J., \& Leitner, S. N. 2006, ApJ, 648, 1043

Fang, T., McKee, C. F., Canizares, C. R., \& Wolfire, M. 2006, ApJ, 644, 174

Fang, T., Sembach, K. R., \& Canizares, C. R. 2003, ApJ, 586, L49

Ferrière, K. 1998, ApJ, 497, 759

Ferrière, K. M. 2001, Rev. Mod. Phys., 73, 1031

Fox, A. J., Savage, B. D., \& Wakker, B. P. 2006, ApJS, 165, 229

Fox, A. J., Savage, B. D., Wakker, B. P., et al. 2004, ApJ, 602, 738

Fox, A. J., Wakker, B. P., Savage, B. D., et al. 2005, ApJ, 630, 332

Fryxell, B., Olson, K., Ricker, P., et al. 2000, ApJS, 131, 273

Galeazzi, M., Gupta, A., Covey, K., \& Ursino, E. 2007, ApJ, 658, 1081

Gardiner, L. T., \& Noguchi, M. 1996, MNRAS, 278, 191

Grcevich, J., \& Putman, M. E. 2009, ApJ, 696, 385

Gupta, A., Galeazzi, M., Koutroumpa, D., Smith, R., \& Lallement, R. 2009, ApJ, 707,644

Henley, D. B., \& Shelton, R. L. 2008, ApJ, 676, 335

Henley, D. B., Shelton, R. L., Kwak, K., Joung, M. R., \& Mac Low, M.-M. 2010, ApJ, 723, 935

Hill, A. S., Haffner, L. M., \& Reynolds, R. J. 2009, ApJ, 703, 1832

Indebetouw, R., \& Shull, J. M. 2004, ApJ, 607, 309

Ito, M., \& Ikeuchi, S. 1988, PASJ, 40, 403

Korpela, E. J., Edelstein, J., Kregenow, J., et al. 2006, ApJ, 644, L163

Kuntz, K. D., \& Snowden, S. L. 2000, ApJ, 543, 195

Kwak, K., Henley, D. B., \& Shelton, R. L. 2011, ApJ, 739, 30 (Paper I)

Kwak, K., \& Shelton, R. L. 2010, ApJ, 719, 523

Lei, S., Shelton, R. L., \& Henley, D. B. 2009, ApJ, 699, 1891

Lockman, F. J., Benjamin, R. A., Heroux, A. J., \& Langston, G. I. 2008, ApJ, 679, L21

Lodders, K. 2003, ApJ, 591, 1220

Maller, A. H., \& Bullock, J. S. 2004, MNRAS, 355, 694

McKernan, B., Yaqoob, T., \& Reynolds, C. S. 2004, ApJ, 617, 232

Meyer, D. M., Cardelli, J. A., \& Sofia, U. J. 1997, ApJ, 490, L103

Meyer, D. M., Jura, M., \& Cardelli, J. A. 1998, ApJ, 493, 222

Mirabel, I. F. 1989, in Structure and Dynamics of the Interstellar Medium, ed. G. Tenorio-Tagle, M. Moles, \& J. Melnick (Lecture Notes in Physics, Vol. 350; Berlin: Springer), 396

Muller, C. A., Oort, J. H., \& Raimond, E. 1963, C. R. Acad. Sci. Paris, 257, 1661

Murphy, E. M., Sembach, K. R., Gibson, B. K., et al. 2000, ApJ, 538, L35

Nicastro, F., Zezas, A., Drake, J., et al. 2002, ApJ, 573, 157

Oegerle, W. R., Jenkins, E. B., Shelton, R. L., Bowen, D. V., \& Chayer, P. 2005, ApJ, 622, 377

Oort, J. H. 1966, Bull. Astron. Inst. Neth., 18, 421

Orlando, S., Bocchino, F., Reale, F., Peres, G., \& Pagano, P. 2008, ApJ, 678, 274

Otte, B., \& Dixon, W. V. D. 2006, ApJ, 647, 312

Park, S.-J., Min, K.-W., Seon, K.-I., et al. 2009, ApJ, 700, 155

Peek, J. E. G., Putman, M. E., McKee, C. F., Heiles, C., \& Stanimirović, S 2007, ApJ, 656, 907

Peek, J. E. G., Putman, M. E., \& Sommer-Larsen, J. 2008, ApJ, 674, 227

Peres, G., Rosner, R., Serio, S., \& Vaiana, G. S. 1982, ApJ, 252, 791

Pettini, M., \& West, K. A. 1982, ApJ, 260, 561

Pietz, J., Kerp, J., Kalberla, P. M. W., et al. 1998, A\&A, 332, 55

Putman, M. E., Thom, C., Gibson, B. K., \& Staveley-Smith, L. 2004, ApJ, 603, L77

R Development Core Team. 2008, R: A Language and Environment for Statistical Computing (Vienna, Austria: R Foundation for Statistical Computing)

Rasmussen, A., Kahn, S. M., \& Paerels, F. 2003, in The IGM/Galaxy Connection, The Distribution of Baryons at $z=0$, ed. J. L. Rosenberg \& M. E. Putman (Dordrecht: Kluwer), 109

Raymond, J. C., \& Smith, B. W. 1977, ApJS, 35, 419

Rosner, R., Tucker, W. H., \& Vaiana, G. S. 1978, ApJ, 220, 643 
Ryu, D., Jones, T. W., \& Frank, A. 2000, ApJ, 545, 475

Sahnow, D. J., Moos, H. W., Ake, T. B., et al. 2000, ApJ, 538, L7

Savage, B. D., \& Massa, D. 1987, ApJ, 314, 380

Savage, B. D., \& Sembach, K. R. 1996, ARA\&A, 34, 279

Savage, B. D., Sembach, K. R., Jenkins, E. B., et al. 2000, ApJ, 538, L27

Savage, B. D., Sembach, K. R., \& Lu, L. 1997, AJ, 113, 2158

Savage, B. D., Sembach, K. R., Wakker, B. P., et al. 2003, ApJS, 146, 125

Sembach, K. R., \& Savage, B. D. 1992, ApJS, 83, 147

Sembach, K. R., Savage, B. D., Shull, J. M., et al. 2000, ApJ, 538, L31

Sembach, K. R., Savage, B. D., \& Tripp, T. M. 1997, ApJ, 480, 216

Savage, B. D., \& Wakker, B. P. 2009, ApJ, 702, 1472

Sembach, K. R., Wakker, B. P., Savage, B. D., et al. 2003, ApJS, 146, 165

Shapiro, P. R., \& Benjamin, R. A. 1993, in Star Formation, Galaxies, and the Interstellar Medium, ed. J. Franco, F. Ferrini, \& G. Tenorio-Tagle (Cambridge: Cambridge Univ. Press), 275

Shelton, R. L. 1998, ApJ, 504, 785

Shelton, R. L. 2006, ApJ, 638, 206

Shelton, R. L., Henley, D. B., \& Dixon, W. V. 2010, ApJ, 722, 302

Shelton, R. L., Sallmen, S. M., \& Jenkins, E. B. 2007, ApJ, 659, 365

Shelton, R. L., Kruk, J. W., Murphy, E. M., et al. 2001, ApJ, 560, 730

Shull, J. M., Stevans, M., Danforth, C., et al. 2011, ApJ, 739, 105

Slavin, J. D., Shull, J. M., \& Begelman, M. C. 1993, ApJ, 407, 83

Smith, R. K., Bautz, M. W., Edgar, R. J., et al. 2007, PASJ, 59, S141

Snowden, S. L., Egger, R., Finkbeiner, D. P., Freyberg, M. J., \& Plucinsky, P. P. 1998, ApJ, 493, 715
Snowden, S. L., Freyberg, M. J., Kuntz, K. D., \& Sanders, W. T. 2000, ApJS, 128,171

Spitzer, L. 1978, Physical Process in the Interstellar Medium (New York: Wiley) Spitzer, L. 1996, ApJ, 458, L29

Stone, J. M., \& Norman, M. L. 1992, ApJ, 390, L17

Thom, C., Peek, J. E. G., Putman, M. E., et al. 2008, ApJ, 684, 364

Tufte, S. L. 2004, in High Velocity Clouds, ed. H. van Woerden, B. P. Wakker, U. J. Schwarz, \& K. S. de Boer (Dordrecht: Kluwer), 167

Wakker, B. P., Savage, B. D., Sembach, K. R., et al. 2003, ApJS, 146, 1

Wakker, B. P., \& van Woerden, H. 1997, ARA\&A, 35, 217

Wakker, B. P., York, D. G., Howk, J. C., et al. 2007, ApJ, 670, L113

Wang, Q. D. 1998, in The Local Bubble and Beyond, ed. D. Breitschwerdt, M. J. Freyberg, \& J. Trümper (Lecture Notes in Physics, Vol. 506; New York: Springer), 503

Wang, Q. D., \& Yu, K. C. 1995, AJ, 109, 698

Weiner, B. J., \& Williams, T. B. 1996, AJ, 111, 1156

Welsh, B. Y., Edelstein, J., Korpela, E. J., et al. 2007, A\&A, 472, 509

Williams, R. J., Mathur, S., Nicastro, F., et al. 2005, ApJ, 631, 856

Wilms, J., Allen, A., \& McCray, R. 2000, ApJ, 542, 914

Wood, B. E., Linsky, J. L., Hébrard, G., et al. 2002, ApJS, 140, 91

Yao, Y., Nowak, M. A., Wang, Q. D., Schulz, N. S., \& Canizares, C. R. 2008, ApJ, $672, \mathrm{~L} 21$

Yao, Y., \& Wang, Q. D. 2007, ApJ, 658, 1088

Yao, Y., Wang, Q. D., Hagihara, T., et al. 2009, ApJ, 690, 143

Yoshino, T., Mitsuda, K., Yamasaki, N. Y., et al. 2009, PASJ, 61, 805

Zsargó, J., Sembach, K. R., Howk, J. C., \& Savage, B. D. 2003, ApJ, 586, 1019 